\title{
Interlayer exciton formation, relaxation, and transport in TMD van der Waals heterostructures
}

\author{
Ying Jiang ${ }^{1}$, Shula Chen ${ }^{1,2}$, Weihao Zheng ${ }^{1}$, Biyuan Zheng ${ }^{1}$ and Anlian Pan ${ }^{1}$
}

\begin{abstract}
Van der Waals ( $\mathrm{vdW}$ ) heterostructures based on transition metal dichalcogenides (TMDs) generally possess a type-II band alignment that facilitates the formation of interlayer excitons between constituent monolayers. Manipulation of the interlayer excitons in TMD vdW heterostructures holds great promise for the development of excitonic integrated circuits that serve as the counterpart of electronic integrated circuits, which allows the photons and excitons to transform into each other and thus bridges optical communication and signal processing at the integrated circuit. As a consequence, numerous studies have been carried out to obtain deep insight into the physical properties of interlayer excitons, including revealing their ultrafast formation, long population recombination lifetimes, and intriguing spinvalley dynamics. These outstanding properties ensure interlayer excitons with good transport characteristics, and may pave the way for their potential applications in efficient excitonic devices based on TMD vdW heterostructures. At present, a systematic and comprehensive overview of interlayer exciton formation, relaxation, transport, and potential applications is still lacking. In this review, we give a comprehensive description and discussion of these frontier topics for interlayer excitons in TMD vdW heterostructures to provide valuable guidance for researchers in this field.
\end{abstract}

\section{Introduction}

Atomically thin transition metal dichalcogenides (TMDs) have received extensive attention due to their unique electronic band structures and the resulting fascinating physical properties ${ }^{1,2}$, such as a direct bandgap in the visible-infrared range, large exciton binding energies of hundreds of $\mathrm{meV}$, and the existence of two intrinsic valley-contrasting quantities, namely, the Berry curvature and the orbital magnetic moment, which allow direct addressing and manipulation of the valley states by external optical, electric, and magnetic fields ${ }^{3-5}$. Furthermore, different TMD monolayers can be vertically stacked to form heterostructures held by weak van der Waals (vdW) forces (Fig. 1a, top panel), which can circumvent the conventional lattice-mismatch problem and

\footnotetext{
Correspondence: Anlian Pan (anlian.pan@hnu.edu.cn)

${ }^{1}$ Key Laboratory for Micro-Nano Physics and Technology of Hunan Province, School of Physics and Electronics, and College of Materials Science and Engineering, Hunan University, Changsha, China

${ }^{2}$ Wuhan National Laboratory for Optoelectronics, Huazhong University of

Science and Technology, Wuhan, China
}

thus significantly expand the family of this kind of heterostructure ${ }^{6}$. Additionally, as a new type of quantum material $^{7,8}$, TMD vdW heterostructures not only combine the already extraordinary properties of the constituent monolayers but also provide a rich platform for exploring new fascinating physics and engineering them by various strategies, such as the material type, crystallographic alignment, stacking sequence, or external field.

One such novel physical phenomenon is the emergence of interlayer excitons. Both theoretical ${ }^{9-14}$ and experimental ${ }^{15-19}$ studies demonstrated that most TMD vdW heterostructures feature a type-II band alignment with the conduction band minimum (CBM) and valence band maximum (VBM) located in different monolayers (Fig. 1a, bottom panel), which facilitates interlayer charge transfer, with electrons accumulating in the layer with the lower $\mathrm{CBM}$ and holes accumulating in the other layer with the higher VBM. Additionally, the atomic layer thickness of the interlayer separation guarantees strong electron-hole Coulomb interactions between adjacent layers with large binding energies, producing spatially separated but bound 


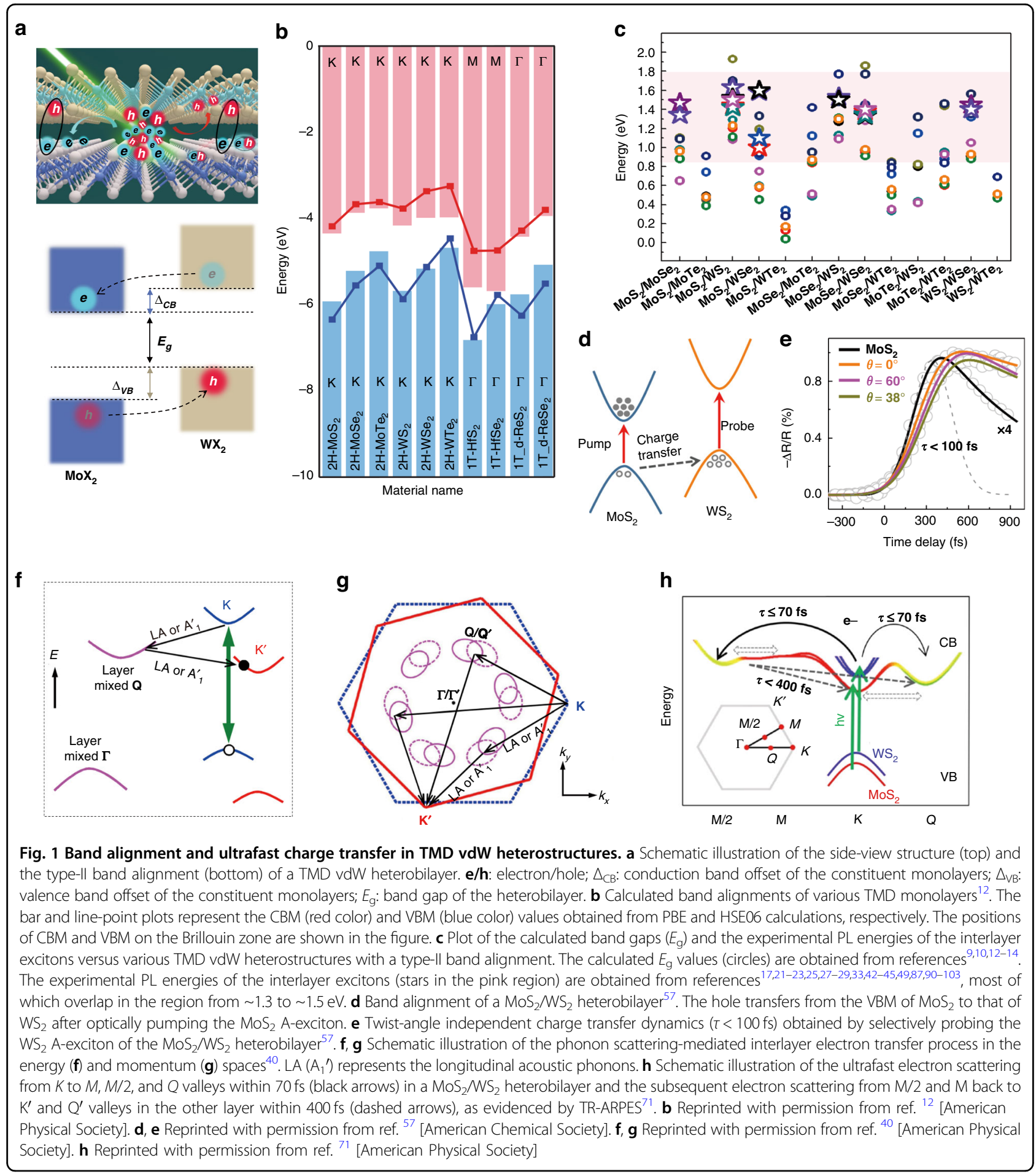

electron-hole pairs, that is, interlayer excitons ${ }^{20-22}$. Because of this spatially indirect nature, the reduced overlap of the electron and hole wavefunctions in interlayer excitons provides them with long lifetimes, reaching hundreds of nanoseconds ${ }^{23-26}$ or even microseconds ${ }^{27}$. In addition, the spatial separation between charges creates a permanent electrical dipole moment in the out-of-plane direction, which allows electrical control of their optical and transport properties along with the generation of repulsive dipole-dipole interactions between them ${ }^{22,28-30}$. All these features make interlayer excitons an appealing platform for exploring many-body effects, such as Bose-Einstein condensation (BEC) and superfluidity ${ }^{31,32}$ and highly desirable for developing potential excitonic 
circuits with long-range exciton transport properties $^{29,30,33,34}$. In addition, the interlayer excitons carry intrinsic valley-contrasting physics inherited from the constituent monolayers, which further enriches fundamental explorations and promising applications with valley functionalities ${ }^{35}$. More intriguingly, the moiré pattern that arises due to the lattice mismatch and rotation angle between the adjacent layers consists of periodically varied local interlayer atomic registries, and is able to create a periodic potential to trap the interlayer excitons with specific optical selective rules, leading to the socalled moiré interlayer excitons, opening up new avenues for quantum manipulation of the quasi-particles towards programmable quantum optics ${ }^{36}$. Apparently, interlayer excitons, as a special excitonic system, bring about a number of fascinating properties that are inaccessible by conventional direct excitons, which lays the basis for exploring novel sciences and developing promising solidstate excitonic devices based on interlayer excitons.

Motivated by these exciting physics from the interlayer excitons in TMD vdW heterostructures, below, we give a systematic overview of interlayer exciton formation, relaxation, transport, and potential applications in excitonic optoelectronic devices. Specifically, the band alignment and ultrafast charge transfer followed by interlayer exciton formation as well as its fundamental properties are first discussed. Moiré interlayer excitons, as a newly emerging popular research topic, are also detailed in this section. Then, the interlayer exciton relaxation processes including the population recombination dynamics, the intervalley scattering process, and the valley-polarized dynamics in TMD vdW heterostructures are reviewed. Later, the interlayer exciton transport under both an electric field and a moiré potential are described. A brief introduction of the recent progress on excitonic optoelectronic devices based on interlayer excitons is given. Finally, we present the conclusions with an outlook on future opportunities for interlayer excitons in TMD-based heterostructures.

\section{Interlayer exciton formation in TMD vdW heterostructures}

In this section, we review the band alignment and charge transfer, interlayer exciton formation, fundamental properties of interlayer excitons, and moiré interlayer excitons in TMD vdW heterostructures.

\section{Band alignment and charge transfer}

The band alignment of semiconductor heterostructures is of central importance in determining their physical properties and potential applications. Three typical types of band alignments (straddling/type-I, staggered/type-II, and broken/type-III) can be constructed in these heterostructures, with their formation being generally understood by Anderson's rule ${ }^{14}$. For TMD vdW heterostructures, numerous theoretical calculations have been performed to study their band structures, and a type-II alignment was found in most combinations of constituted monolayers (Fig. 1b, c) ${ }^{9-14}$, with the CBM and VBM residing in opposite monolayers. Experimental determination of the band alignments was also carried out and revealed that a staggered form was the prevailing configuration in representative TMD heterostructures. For instance, a type-II alignment with a valence band (VB) offset of $0.83 \mathrm{eV}$ and a conduction band $(\mathrm{CB})$ offset of $0.76 \mathrm{eV}$ was found in the $\mathrm{MoS}_{2} / \mathrm{WSe}_{2}$ heterostructure by using microbeam X-ray photoelectron spectroscopy ( $\mu$-XPS) and scanning tunneling microscopy/spectroscopy $(\mathrm{STM} / \mathrm{STS})^{15}$. Similarly, a type-II alignment in the $\mathrm{MoS}_{2} / \mathrm{MoTe}_{2}$ heterostructure was measured by using high-resolution XPS and UV-Vis absorption spectroscopy, with a reported $\mathrm{VB}$ offset of $0.9 \mathrm{eV}$ and a CB offset of $0.46 \mathrm{eV}^{19}$. The $\mathrm{VB}$ offset of $0.3 \mathrm{eV}$ in the $\mathrm{MoSe}_{2} / \mathrm{WSe}_{2}$ heterostructure was also experimentally determined by using submicrometer angle-resolved photoemission spectroscopy ( $\mu$-ARPES) ${ }^{17}$. Additionally, the interlayer hybridization, which is sensitive to the interlayer coupling strength (and thus the interlayer distance and twist angle between the constituent monolayers), can affect the band type of TMD vdW heterostructures. Both theoretical $^{37-41}$ and experimental ${ }^{15,17,18,22,42,43}$ results showed that the VBM and CBM of most studied vdW heterostructures favored retention at the $K$ valleys in opposite layers to form a direct band gap, with negligible or weak interlayer hybridization near these valleys. However, in the $\Gamma$ and $\Lambda$ (or $Q$ ) valleys, significant interlayer hybridization was found with an interlayer coupling strength of several hundred $\mathrm{meV}$, which was comparable to the band offset $^{40}$. Such strong interlayer coupling can lead to large energy shifts and may move the VBM/CBM of the heterostructures to the $\Gamma / \Lambda$ (or $Q$ ) valleys, thus forming an indirect type of band gap in some cases ${ }^{43-45}$, similar to what was observed in homobilayers and bulk TMDs ${ }^{17,46,47}$. In addition to influences from the large differences in band offsets at various valleys, the difference in the orbital characteristics was considered an important factor for the resulting variant interlayer hybridization in momentum space $^{17,48}$. It was reported that the bands at $K$ valleys generally featured an in-plane orbital character, while those at $\Gamma / \Lambda$ (or $Q$ ) valleys were characterized by an out-ofplane orbital character and therefore were more sensitive to the interlayer interactions for orbital hybridization ${ }^{48}$. Hence, the interlayer distance and twist angle (or stacking manner), which affect the interlayer interactions and/or the interlayer coupling strength, could substantially modify the bands at the $\Gamma / \Lambda$ (or $Q$ ) valleys. This was supported by the fact that the energies of the $\Gamma$ and $\Lambda$ (or Q) points depended sensitively on the interlayer distance and stacking manner ${ }^{37,42,49-51}$. 
Following both theoretical predictions and experimental determination of a type-II band alignment in most TMD vdW heterostructures, charge transfer as a natural outcome of this configuration spurred research interest in these two-dimensional (2D) heterostructures ${ }^{52-54}$. The first experimental observation of ultrafast charge transfer was reported in a $\mathrm{MoS}_{2} / \mathrm{WS}_{2}$ heterostructure using both photoluminescence (PL) mapping and femtosecond pump-probe spectroscopy ${ }^{52}$. This demonstrated that holes in the $\mathrm{MoS}_{2}$ monolayer could efficiently transfer into the $\mathrm{WS}_{2}$ monolayer within $50 \mathrm{fs}$ after photoexcitation. Then, both electron and hole transfer in opposite directions on a sub-picosecond time scale were evidenced in the $\mathrm{MoS}_{2} / \mathrm{MoSe}_{2}$ heterostructure ${ }^{53}$. After these initial works, charge transfer dynamics in TMD vdW heterostructures have been extensively investigated, from which the interfacial charge transfer was found to be universally ultrafast (mostly within $100 \mathrm{fs}$ ) and twist-angle independent (Fig. 1d, e) ${ }^{42,55-63}$. These observations are very puzzling because (1) the interlayer van der Waals coupling in heterostructures is normally much weaker than the intralayer covalent bonding, and hence, the interlayer charge transfer is not expected to be so rapid as compared to the intralayer exciton dynamics; and (2) momentum mismatch in displaced $\pm \mathrm{K} / \pm \mathrm{K}^{\prime}$ valleys is inevitable due to the lattice mismatch and twist-angle between the constituent monolayers (note that " $+\mathrm{K}$ " and " $-K^{\prime}$ " (or " $+K^{\prime \prime}$ " and " $-K^{\prime \prime}$ ) indicate the opposite valleys of the same monolayer, while " $+\mathrm{K}$ " and " $+\mathrm{K}^{\prime \prime}$ " (or " $-\mathrm{K}$ " and " $-K^{\prime \prime}$ ) describe the same $K$ valley but in the opposite monolayers. The same applies to other valleys such as the $Q$ and $\Gamma$ valleys described below), so how can the interlayer charge transfer be so effective and twist-angle independent, regardless of the crystal orientation or momentum mismatch?

A number of studies have been carried out to explore the intrinsic mechanism for efficient charge transfer in TMD vdW heterostructures ${ }^{38,40,64-72}$. In TMD monolayers, one may find that the exciton binding energy $(0.5-1 \mathrm{eV})$ is comparable to the typical Frenkel exciton ${ }^{73-77}$, while its wavefunction favors a Wannier-Mott type with electron-hole separation extending over several tens of unit cells (the exciton Bohr radius was calculated to be $\sim 1-3 \mathrm{~nm})^{73,78,79}$. Therefore, for TMD vdW heterostructures, although the constituent monolayers are in contact with weak vdW coupling, the layer separation is less than $1 \mathrm{~nm}$, and the layer-separated electrons and holes can still undergo strong Coulomb interactions to form bound exciton states ${ }^{2,35,52}$ (i.e., interlayer excitons, which are discussed later). These bound exciton states are thought to be energetically favorable and can compete with the intralayer exciton states ${ }^{52}$. That is, the photoexcited electrons and holes should have comparable probability to form these layer-separated bound exciton states in addition to the intralayer exciton states, which probably accounts for the efficient interlayer charge transfer in vdW heterostructures ${ }^{52}$. For the observed twist-angle independence of charge transfer ${ }^{56-58}$, a very likely mechanism is that phonon scattering coupled with interlayer hybridization circumvents the momentum mismatch for such rapid charge transfer ${ }^{40}$. As proposed (Fig. 1f, g) ${ }^{40}$, interlayer charge transfer can efficiently take place via two sequential steps: first, a photoexcited electron is scattered from the $K$ valley to the strongly layer hybridized $Q / Q^{\prime}$ valley $\left(\Gamma / \Gamma^{\prime}\right.$ valley for a hole) through the emission of an intralayer phonon, and then it subsequently relaxes from the $Q^{\prime}$ valley ( $\Gamma^{\prime}$ valley for a hole) to the $K^{\prime}$ valley in the opposite layer by emitting another phonon. The interlayer charge transfer via $Q / Q^{\prime}$ or $\Gamma / \Gamma^{\prime}$ valleys is expected to be rapid ( $<50 \mathrm{fs})$ due to the strong interlayer coupling or hybridization in these regions. Moreover, the $\Gamma$ positions with strong layer mixing are not affected by the interlayer twist, and the $Q$ valleys are always on a ring region with strong interlayer coupling for any twist angle (Fig. 1g), both of which explain the observed twist-angle independence of the charge transfer well. This proposed mechanism was recently experimentally supported in a $\mathrm{MoS}_{2} / \mathrm{WS}_{2}$ heterobilayer with timeresolved and angle-resolved photoemission spectroscopy $(\mathrm{TR}-\mathrm{ARPES})^{71}$, where the ultrafast scattering of electrons from the $K$ to $M, M / 2$, and $Q$ valleys was evidenced within $70 \mathrm{fs}$, followed by electron scattering from $M / 2$ and $M$ back to the $K^{\prime}$ and $Q^{\prime}$ valleys in the other layer within $400 \mathrm{fs}$ (Fig. 1h). These studies suggest that $\Gamma$ and $Q$ valleys with strong interlayer hybridization play an important role in mediating ultrafast and twist-insensitive charge transfer in TMD vdW heterostructures.

\section{Interlayer exciton formation}

As stated above, immediately after rapid interlayer charge transfer, a strong Coulomb interaction of the electrons and holes in opposite layers could exist due to the layer separation $(\sim 0.7 \mathrm{~nm})$ comparable to the intralayer exciton Bohr radius $(\sim 1-3 \mathrm{~nm})^{73,78,79}$, which facilitates the formation of interlayer excitons that have been both theoretically $^{80}$ and experimentally evidenced ${ }^{20-22,42}$. The binding energy of the interlayer exciton, with a reported value of $\sim 100-350 \mathrm{meV}$ from both theoretical ${ }^{81-86}$ and experimental ${ }^{17,21,42,55,87-89}$ results, is a further indication of the strong Coulomb interaction strength between the layer-separated electrons and holes, of which the value depends sensitively on the interlayer distance ${ }^{83,85}$.

The resulting interlayer excitons feature the lowest energy configuration, with the electrons and holes residing in the CBM and VBM of the opposite layers due to the type-II band alignment. Hence, the hallmark of forming an interlayer exciton is the appearance of an extra PL peak at a lower energy along with PL quenching of the 

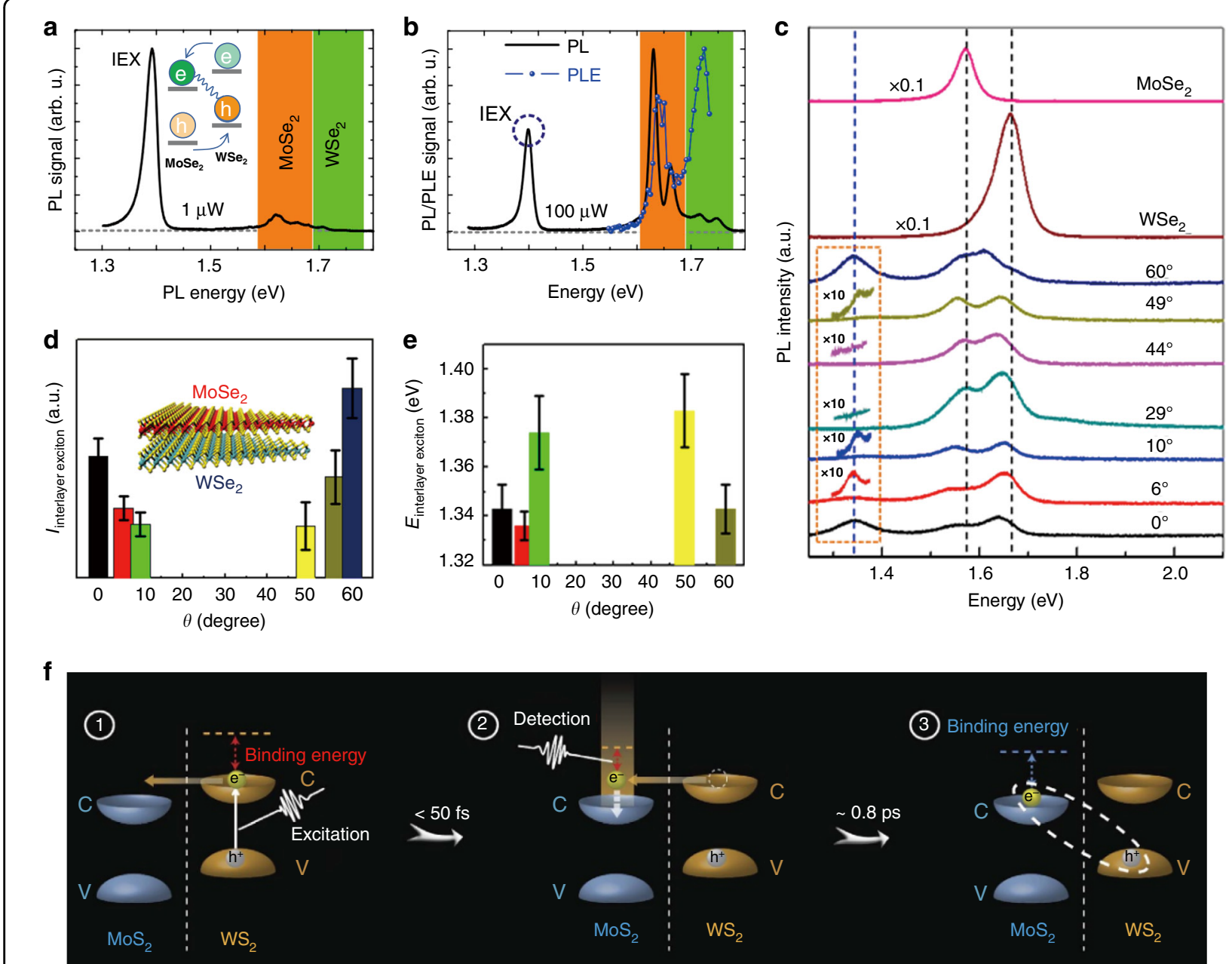

Fig. 2 Interlayer exciton formation in TMD vdW heterostructures. a PL spectrum of the $\mathrm{MoSe}_{2} / \mathrm{WSe}_{2}$ heterostructure measured at $4.5 \mathrm{~K}$ with $1 \mu \mathrm{W}$ excitation power ${ }^{28}$. IEX represents the emissions from interlayer excitons. $\mathbf{b} \mathrm{PL}$ spectrum of the $\mathrm{MoSe}_{2} / \mathrm{WSe}_{2}$ heterostructure (black line) measured at $4.5 \mathrm{~K}$ with $100 \mu \mathrm{W}$ excitation power and the PLE spectrum (blue dots) of the interlayer exciton emission (IEX, marked by the blue dashed circle) ${ }^{28}$. c PL spectra of the $\mathrm{MoSe}_{2}$ monolayer, WSe $\mathrm{W}_{2}$ monolayer, and $\mathrm{MoSe}_{2} / \mathrm{WSe}_{2}$ heterobilayer for various twist angles $\left(0^{\circ} \leq \theta \leq 60^{\circ}\right)$ at room temperature ${ }^{101}$. d, e Twist-angle-dependent PL intensity (d) and energy (e) of the interlayer excitons formed in $\mathrm{MoSe}_{2} / \mathrm{WSe}_{2}$ heterobilayers ${ }^{101}$. f Illustration of the interlayer exciton formation process in $\mathrm{MoS}_{2} / \mathrm{WS}_{2}$ heterostructures as revealed by transient absorption spectroscopy ${ }^{55}$. a, b Reprinted with permission from ref. ${ }^{28}$ [IOP Publishing]. c-e Reprinted with permission from ref. ${ }^{101}$ [American Chemical Society]. f Reprinted with permission from ref. ${ }^{55}$ [Springer Nature Limited]

constituent monolayers (Fig. 2a), which has been observed in various representative TMD vdW heterostructures such as $\mathrm{WS}_{2} / \mathrm{WSe}_{2}{ }^{58,90,91}, \mathrm{MoS}_{2} / \mathrm{MoSe}_{2}{ }^{53,87,92}, \mathrm{MoS}_{2} /$ $\mathrm{WS}_{2}{ }^{42,43,49,55,93,94}, \quad \mathrm{MoS}_{2} / \mathrm{WSe}_{2}{ }^{20,21,29,44,95-97}, \quad \mathrm{MoSe}_{2} /$ $\mathrm{WS}_{2}{ }^{98-100}, \quad \mathrm{MoSe}_{2} / \mathrm{WSe}_{2}{ }^{17,22,23,25,27,28,33,45,101-104}$, and $\mathrm{MoS}_{2} / \mathrm{MoSe}_{2} / \mathrm{MoS}_{2}{ }^{24,105}$ and also in homobilayers ${ }^{46,106}$, with the PL energy of interlayer excitons ranging from $\sim 1.0$ to $\sim 1.6 \mathrm{eV}$ (Fig. 1c). PL excitation (PLE) measurement is an alternative tool for supporting interlayer exciton formation ${ }^{22,24,28}$. As observed (Fig. 2b) ${ }^{28}$, the PL emission of interlayer excitons only emerges when the laser energy is high enough to excite the monolayer with a narrower band gap, and the PL intensity is resonantly enhanced when the laser energy matches the A-exciton resonance of each monolayer, suggesting the interlayer nature of such an emission. The formation dynamics of the interlayer excitons have also been revealed by various transient spectroscopies ${ }^{55,89}$. In a $\mathrm{MoS}_{2} / \mathrm{WS}_{2}$ heterostructure $^{55}$, the interlayer exciton formation process was reported to include two steps (Fig. 2f): first, an intermediate hot interlayer exciton state with a binding energy $<0.17 \mathrm{eV}$ was initially formed within $50 \mathrm{fs}$, and second, it relaxed to a tightly bound interlayer exciton state within $\sim 800 \mathrm{fs}$ featuring a binding energy $>0.36 \mathrm{eV}$. However, a recent work demonstrated that the interlayer exciton state in the $\mathrm{WS}_{2} / \mathrm{WSe}_{2}$ heterostructure ${ }^{89}$, with a binding energy of $\sim 0.13 \mathrm{eV}$, was formed within $100 \mathrm{fs}$ and directly transformed from the intralayer exciton gas without a 
pronounced intermediate hot state. The origin of these discrepancies in the formation process of interlayer excitons needs to be further explored.

Note that interlayer excitons are not always formed or observed in vdW heterostructures. Their emergence requires certain prerequisites such as appropriate interlayer distance and momentum mismatch, both of which could significantly affect the interlayer coupling between the constituent monolayers. The interlayer distance of the heterobilayers is generally improved by thermal annealing $^{49,92,100}$, and can be controlled by inserting insulate layers (i.e., $\mathrm{hBN}$ ) between the TMD monolayers ${ }^{20,107}$. The momentum mismatch is predominantly influenced by the lattice mismatch and twist angle of the opposite monolayers. In contrast to the interlayer charge transfer, both the PL intensity and energy of the interlayer excitons were demonstrated to have twist-angle dependence ${ }^{41,42,95,100,101}$. For instance, in the $\mathrm{MoSe}_{2} / \mathrm{WSe}_{2}$ heterostructures ${ }^{101}$, the PL intensity of the interlayer excitons was enhanced at angles near $0^{\circ}$ and $60^{\circ}$ but disappeared at other intermediate angles $\left(10-50^{\circ}\right)$, and their PL energies also varied with the twist angle (Fig. 2c-e), which was attributed to the twist-angle dependence of the interlayer coupling strength in these heterostructures. In addition, it was reported that the PL energy of interlayer excitons could be tuned by many other factors such as the vdW bandgap ${ }^{90}$, electric and magnetic fields ${ }^{22,108-110}$, cavity $^{11-113}$, pressure $^{114}$, and layer number ${ }^{115}$. Moreover, even electroluminescence (EL) from interlayer excitons was observed in TMD vdW heterobilayers under a forward bias ${ }^{30,32,102}$.

\section{Fundamental properties of interlayer excitons}

An interlayer exciton is an exotic exciton system that is distinguished from an intralayer exciton and possesses various novel and intriguing properties, such as the existence of a reduced transition dipole and a static electric dipole as well as inherited valley-contrasting physics from monolayers. All these properties are of central interest in both fundamental physics and potential applications, as discussed below.

\section{Reduced transition dipole}

Due to the spatially indirect nature of the interlayer exciton, its transition dipole was theoretically predicted to be one to two orders of magnitude smaller than that of the intralayer exciton ${ }^{80,116}$, and therefore, the corresponding oscillator strength is expected to be dramatically reduced with respect to that of an intralayer exciton. This is supported by an experimental study of the photocurrent measurements of interlayer excitons in a $\mathrm{MoSe}_{2} / \mathrm{WSe}_{2}$ heterobilayer ${ }^{102}$, where the photocurrent amplitude from the interlayer excitons was shown to be approximately 200 times smaller than that of the intralayer excitons, meaning that the interlayer exciton oscillator strength was two orders of magnitude smaller than that of the intralayer excitons due to the spatial separation of the electrons and holes in the opposite layers. In addition, the transition dipole of the interlayer excitons was shown to be quite sensitive to the kinematical momentum sum $(\mathbf{Q})$ of the electrons and holes in the opposite monolayers, leading to so-called light cones with an ordered hexagonal array in momentum space (Fig. 3a-c) ${ }^{35,80}$. As shown in Fig. 3b, an interlayer exciton consists of an electron situated at the momentum position $\tau^{\prime} \mathbf{K}^{\prime}+\mathbf{k}^{\prime}$ and a hole at $\tau \mathbf{K}-\mathbf{k}\left(\tau^{\prime}\right.$ and $\tau$ are the electron and hole valley indices $)^{35,80}$. The kinematical momentum $(\mathbf{Q})$ of the interlayer exciton (or the kinematical momentum sum of the electron and hole) was defined as $\mathbf{Q} \equiv \mathbf{k}^{\prime}+\mathbf{k}$ by previous reports ${ }^{35,80}$. For an interlayer exciton with zero kinematical momentum $(\mathbf{Q}=$ 0 ), the electron and hole are located separately in the CBM $\left(\tau^{\prime} \mathbf{K}^{\prime}\right)$ and $\operatorname{VBM}(\tau \mathbf{K})$ with uncompensated momentum mismatch due to the unavoidable lattice mismatch and/or interlayer twist between the layers, and in this case, the interlayer exciton is considered to be optically dark with a vanished transition dipole and forbidden direct radiative recombination; namely, the interlayer exciton is out of the light cone. However, for an interlayer exciton with a certain kinematical momentum $\mathbf{Q}$ by which the momentum mismatch between the electron and hole can be compensated (that is, $\mathbf{Q}=\tau \mathbf{K}-\tau^{\prime} \mathbf{K}^{\prime}$ ), in this case, the interlayer exciton possesses a finite transition dipole for the direct optical transition and thus is optically bright; namely, the interlayer exciton is within the light cone. In addition, the transition dipole strength of a light cone was reported to decay quickly with the magnitude of the momentum ${ }^{35,80}$, so the most bright light cones are those at $\mathbf{Q}$ values nearest to $\mathbf{Q}=0$, and other light cones are Umklapp type (Fig. 3b), which is consistent with experimental results showing that the interlayer excitons can only be observed in heterostructures with relatively small interlayer distances and limited rotational angles for the finite interlayer coupling between layers. Compared to an intralayer exciton, a significant merit of the reduced transition dipole of an interlayer exciton is its substantially extended recombination and valley polarization lifetimes due to the reduced overlap of the electron and hole wavefunctions, which inspires research exploring spinelectronic, valley-electronic, and optoelectronic physics and applications based on TMD vdW heterostructures.

\section{Static electric dipole}

Since electrons and holes are confined in opposite layers of a type-II vdW heterostructure, interlayer excitons have a static electric dipole along the out-of-plane direction $(p=$ $e \cdot d$, where $e$ is the charge quantity and $d$ is the charge separation distance), which allows their energy to be tuned $(\Delta \varepsilon)$ by an external electric field $(E)$ along the dipole axis (i.e., the Stark effect, $\Delta \varepsilon=-p \cdot E)^{22,95,117}$. Strong and linear 


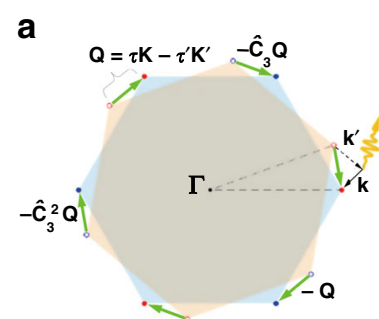

C

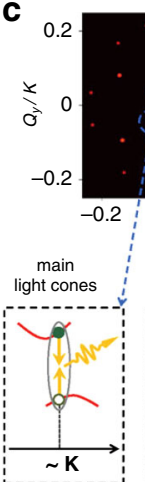

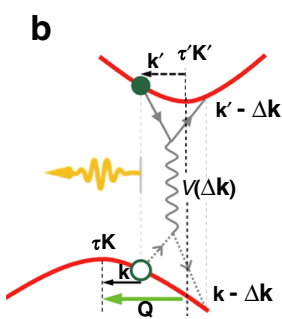

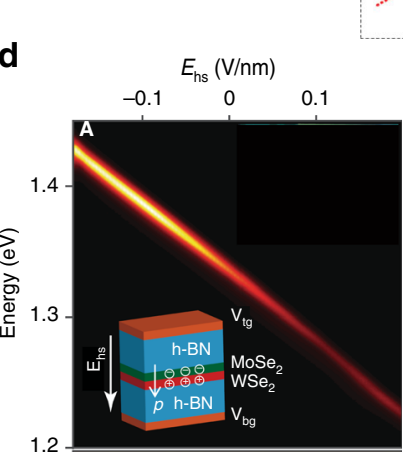

$\mathbf{f}$

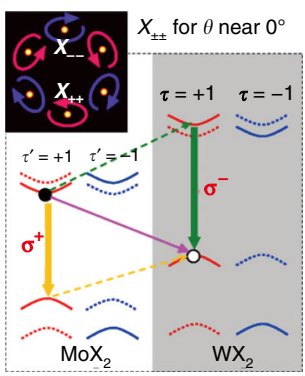

e

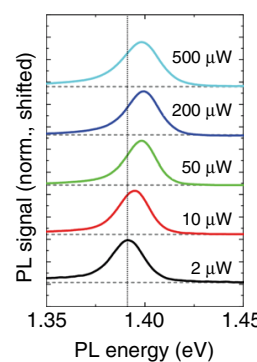

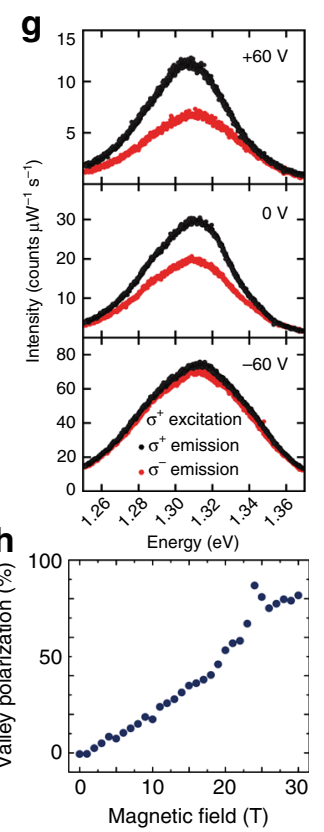

Fig. 3 Fundamental properties of interlayer excitons. a Brillouin zone corners of the $W X_{2}$ layer ( $\left(\mathbf{K}\right.$, the solid dots) and the MoX $X_{2}$ layer ( $\tau^{\prime} \mathbf{K}^{\prime}$, the open dots) with a small twist and/or lattice mismatch ${ }^{80}$. The green arrow represents the displacement vector between the $\tau \mathbf{K}$ and $\tau^{\prime} \mathbf{K}^{\prime}$ corners of the constituent layers. $\hat{C}_{3}$ is the three-fold rotational symmetry. $\mathbf{b}$ The electron-hole interlayer Coulomb interaction $(V(\Delta \boldsymbol{k}))$ conserves their kinematical momentum sum $\mathbf{Q}^{80}$. The interlayer exciton with a certain kinematical momentum $\mathbf{Q}$ equaling the momentum mismatch between the electron and hole $\left(\mathbf{Q}=\mathbf{T} \mathbf{K}-\mathbf{T}^{\prime} \mathbf{K}^{\prime}\right.$, the green arrows) can recombine to emit a photon. $\mathbf{c}$ The main, first-Umklapp, and second-Umklapp light cones in $\mathbf{Q}$ space for $\mathrm{MoSe}_{2} / \mathrm{WSe}_{2}$ heterobilayers with twist angles $\theta$ near $0^{\circ}$ or $60^{\circ 80}$. $\mathbf{d}$ PL energy of the interlayer exciton versus the electric field $\left(E_{\mathrm{hs}}\right)$ applied on the $\mathrm{MoSe}_{2} / \mathrm{WSe}_{2}$ heterostructure ${ }^{30}$. The inset schematically displays the heterostructure cross-section. The white arrows represent the directions of the electric field $\left(E_{h s}\right)$ and the static electric dipole $(p)$. e PL spectra of the interlayer excitons in $\mathrm{MoSe}_{2} / \mathrm{WSe}_{2}$ heterostructures measured at $4.5 \mathrm{~K}$ as a function of excitation power ${ }^{28}$. $\mathbf{f}$ Theoretically predicted valley-dependent elliptically polarized optical selection rules for interlayer excitons $\left(X_{--,} X_{++}\right)$ in the six main light cones ${ }^{80}$. The dipole transition (interlayer hopping) is denoted by solid (dashed) arrows. The valley indices $\left(\tau, \tau^{\prime}\right)$ correspond to $(+,+)$ or $(-,-)$ for $\mathrm{MoX}_{2} / \mathrm{WX}_{2}$ heterobilayers with twist angle $\theta$ near $0^{\circ}$. $\mathbf{g}$ Circular polarization-resolved PL spectra of the interlayer exciton at selected gate voltages. All the data were obtained under $\sigma+$ circularly polarized light excitation, with the co-polarized $(\sigma+)$ and cross-polarized $(\sigma-)$ PL spectra shown in black and red, respectively ${ }^{33}$. $\mathbf{h}$ Magnetic-field-dependent valley polarization of the interlayer exciton ${ }^{25}$. $\mathbf{a}-\mathbf{c}$, $\mathbf{f}$ Reprinted with permission from ref. ${ }^{80}$ [American Physical Society, Springer Nature Limited]. d Reprinted with permission from ref. ${ }^{30}$ [American Association for the Advancement of Science]. e Reprinted with permission from ref. ${ }^{28}$ [IOP Publishing]. g Reprinted with permission from ref. ${ }^{33}$ [American Association for the Advancement of Science]. $\mathbf{h}$ Reprinted with permission from ref. ${ }^{25}$ [Springer Nature Limited]

tuning of the interlayer exciton PL energy with an applied electric field was indeed observed in several studies with a tuning range of $\sim 80-138 \mathrm{meV}^{22,26,30,86,95,117}$, reflecting that the linear Stark effect mainly contributed to the energy shift (Fig. 3d). From the linear fit of the energy shift versus electric field, the dipole size or charge separation $(d)$ has been estimated to be $\sim 0.5-0.8 \mathrm{~nm}$, matching well with the expected layer separation $(\sim 0.7 \mathrm{~nm})^{30,95,117}$. Another consequence of the static electric dipole is the resulting dipole-dipole repulsive interactions between the interlayer excitons $^{22,28,30,118,119}$, as revealed by a blue-shift in the PL energy with increasing exciton density (excitation power) (Fig. 3e $)^{22,28,30}$. Such repulsive interactions owing to the static electric dipole, combined with the ultralong lifetimes due to the reduced transition dipole, make interlayer excitons a highly promising platform for exploring excitonic Bose-Einstein condensate (BEC) and superconductivity phenomena ${ }^{31,32,120}$.

\section{Valley-contrasting physics}

The valley pesudospins in TMD monolayers are known to be accessible and controllable by external optical fields (via valley-dependent optical selection rules) ${ }^{121-124}$, electric fields (via the valley Hall effect) ${ }^{125-128}$, and magnetic fields (via the valley Zeeman effect) ${ }^{129,130}$ owing to the existence of intrinsic valley-contrasting physical quantities (namely, the Berry curvature and orbital magnetic moment) to distinguish the $\pm K$ valleys $^{131-133}$. It was reported that such valley-contrasting physics were inherited by the interlayer excitons in TMD vdW heterostructures $^{80,104}$. Theories predicted that interlayer excitons in the light cones have valley-dependent optical selection rules with opposite helicities for opposite valleys (Fig. 3f ${ }^{80,134}$, which was experimentally evidenced in circularly polarized PL measurements with apparent valley polarization for the interlayer excitons ${ }^{24,27,33,45,117}$. For instance, a valley polarization up to $80 \%$ was reported for 
interlayer excitons in a $\mathrm{MoSe}_{2} / \mathrm{WSe}_{2}$ heterostructure (without externally applied electric or magnetic fields) ${ }^{135}$. Such high valley polarization of interlayer excitons implies efficient interlayer charge transfer accompanied by conserved spin-valley polarization transfers, as revealed in the $\mathrm{MoSe}_{2} / \mathrm{WSe}_{2}$ heterostructure, with robust spin-valley conserved transfers for different twist angles ${ }^{104}$. Additionally, the valley polarization of interlayer excitons can be manipulated by external electric and magnetic fields, with the polarization degree varying with the applied field (Fig. 3g, h) 25,27,33,110,117. Moreover, both valley Hall and Zeeman effects were observed for interlayer excitons $^{25,110,136}$, similar to those for intralayer excitons. All of these results indicate that the valley-contrasting physics in monolayers is successfully inherited by the interlayer excitons. However, note that circularly polarized PL from interlayer excitons with a helicity opposite to the optical excitation was also observed in TMD vdW heterostructures $^{24,137}$, implying a reversed polarization selection rule for interlayer excitons in certain cases. In addition, due to the spin-orbit splitting of the conduction band, spin-singlet and spin-triplet interlayer excitons with opposite helicities in the same valley configuration and atomic registry were both theoretically predicted ${ }^{134}$ and experimentally identified ${ }^{110,135}$ in TMD vdW heterostructures, suggesting very rich but also complicated optical properties for the interlayer excitons.

Note that the magnetic dipole of the interlayer exciton is also quite different from that of the intralayer exciton, as manifested by their distinct $g$ factors. An effective $g$ factor of -15 was reported for the interlayer excitons in a $\mathrm{MoSe}_{2} / \mathrm{WSe}_{2}$ heterostructure with a twist angle of $\sim 54^{\circ}$, which is much larger than that of intralayer excitons in TMD monolayers (approximately -4 in most cases ${ }^{138-140}$ ), thus producing giant valley Zeeman splitting of the interlayer excitons with near-unity valley polarization under magnetic fields ${ }^{25}$. A recent study also demonstrated large $g$ factors for both spin-singlet $(\sim 10.7)$ and spintriplet $(\sim 15.2)$ interlayer excitons in a $\mathrm{MoSe}_{2} / \mathrm{WSe}_{2}$ heterostructure with a $60^{\circ}$ twist angle ${ }^{110}$. Moreover, the $g$ factor of the interlayer excitons was found to be stackingdependent. For instance, the $g$ factors of interlayer excitons in $\mathrm{MoSe}_{2} / \mathrm{WSe}_{2}$ heterostructures with twist angles of $2^{\circ}$ and $57^{\circ}$ were determined to be approximately 6.7 and -15.9 , respectively ${ }^{141}$. Intriguingly, the $g$ factor of interlayer excitons in $\mathrm{MoSe}_{2} / \mathrm{WSe}_{2}$ heterostructures with a twist angle approaching $60^{\circ}$ (H-type configuration) was always larger than that with a twist angle approaching $0^{\circ}$ (R-type configuration) $)^{25,110,117,141,142}$, and the corresponding sign was opposite for the two stacking configurations ${ }^{141}$. These experimental findings were further revealed in a recent theoretical study ${ }^{143}$. Larger effective $g$ factors with opposite signs were theoretically predicted for the interlayer excitons in the H-type $\left(60^{\circ}\right) \mathrm{MoSe}_{2} /$
$\mathrm{WSe}_{2}$ heterostructure ${ }^{143}$ than for those in the R-type $\left(0^{\circ}\right)$ system, which matched the experimental results well. The differences of $g$ values and their signs between the R-type and $\mathrm{H}$-type heterostructures were interpreted as distinct valley pairings for the conduction and valence bands in the two systems ${ }^{141,143}$. In the $\mathrm{H}$-type system, the valley indexes for the conduction and valence bands were opposite $\left(\left(+\mathrm{K},-\mathrm{K}^{\prime}\right)\right.$ or $\left.\left(-\mathrm{K},+\mathrm{K}^{\prime}\right)\right)$, while those in the Rtype system were the same $\left(\left(+\mathrm{K},+\mathrm{K}^{\prime}\right)\right.$ or $\left.\left(-\mathrm{K},-\mathrm{K}^{\prime}\right)\right)$. This consequently produced a larger effective $g$ factor in the H-type system (but with the opposite sign), when the $g$ factor of the conduction band minus that of the valence band ${ }^{141,143}$. In addition, the theoretical analysis showed that the spin-flip transitions could provide an extra spin contribution to the $g$ factor, leading to the effective $g$ factor for the spin-triplet interlayer excitons being higher than that for spin-singlet interlayer excitons ${ }^{143}$, which is consistent with the above experimental results ${ }^{110}$. These studies demonstrate that the $g$ factor of interlayer excitons in TMD vdW heterostructures is both spindependent and stacking-dependent, and most importantly, that the measured $g$ values and their signs can serve as a valuable basis for determining the nature of the interlayer excitons (such as spin-singlet or spin-triplet and R-type or H-type).

\section{Moiré interlayer excitons}

Intrigued by the amazing discovery of Mott insulating states and unconventional superconductivity in magicangle twisted graphenes ${ }^{144,145}$, significant interest has been ignited in twisted $2 \mathrm{D} \mathrm{vdW}$ heterobilayers featuring moiré patterns or superlattices ${ }^{18,36,38,41,47,116,134,141,146-156}$, which has greatly flourished in the new research area called "twistronics". For TMD vdW heterobilayers with a small lattice mismatch and/or rotational misalignment, a moiré pattern could be formed naturally with periodic changes in the interlayer atomic registry, and the corresponding moiré period varying from several nanometers to tens of nanometers is given by $b \approx a / \sqrt{\delta^{2}+\theta^{2}}$, where $a$ is the monolayer lattice constant, $\delta$ is the lattice mismatch, and $\theta$ is the relative twist angle ${ }^{36,116}$. In such a moiré superlattice, the local interlayer atomic registry varies continuously with three high-symmetry sites (A, B, and C) preserving the three-fold rotational symmetry $\hat{C}_{3}$ (Fig. $4 \mathrm{a}, \mathrm{b})^{150}$, which are typically denoted by $\left(R_{\mathrm{h}}^{\mathrm{h}}, R_{\mathrm{h}}^{\mathrm{X}}, R_{\mathrm{h}}^{\mathrm{M}}\right)$ or $\left(H_{\mathrm{h}}^{\mathrm{h}}, H_{\mathrm{h}}^{\mathrm{X}}, H_{\mathrm{h}}^{\mathrm{M}}\right)(X$ : chalcogen site, $M$ : metal site, and $h$ : hollow center of the hexagon) for R-type or H-type stacked heterobilayers. $R_{\mathrm{h}}^{\mathrm{u}}\left(H_{\mathrm{h}}^{\mathrm{u}}, u=X, M, h\right)$ means that the $u$ site of the electron layer overlaps vertically with the $h$ site of the hole layer. As reported, this alteration of the local interlayer atomic registry across a moiré superlattice could consequently lead to lateral modulation of both the interlayer distance and the local band gap, producing a position-dependent potential (i.e., moiré potential) for 

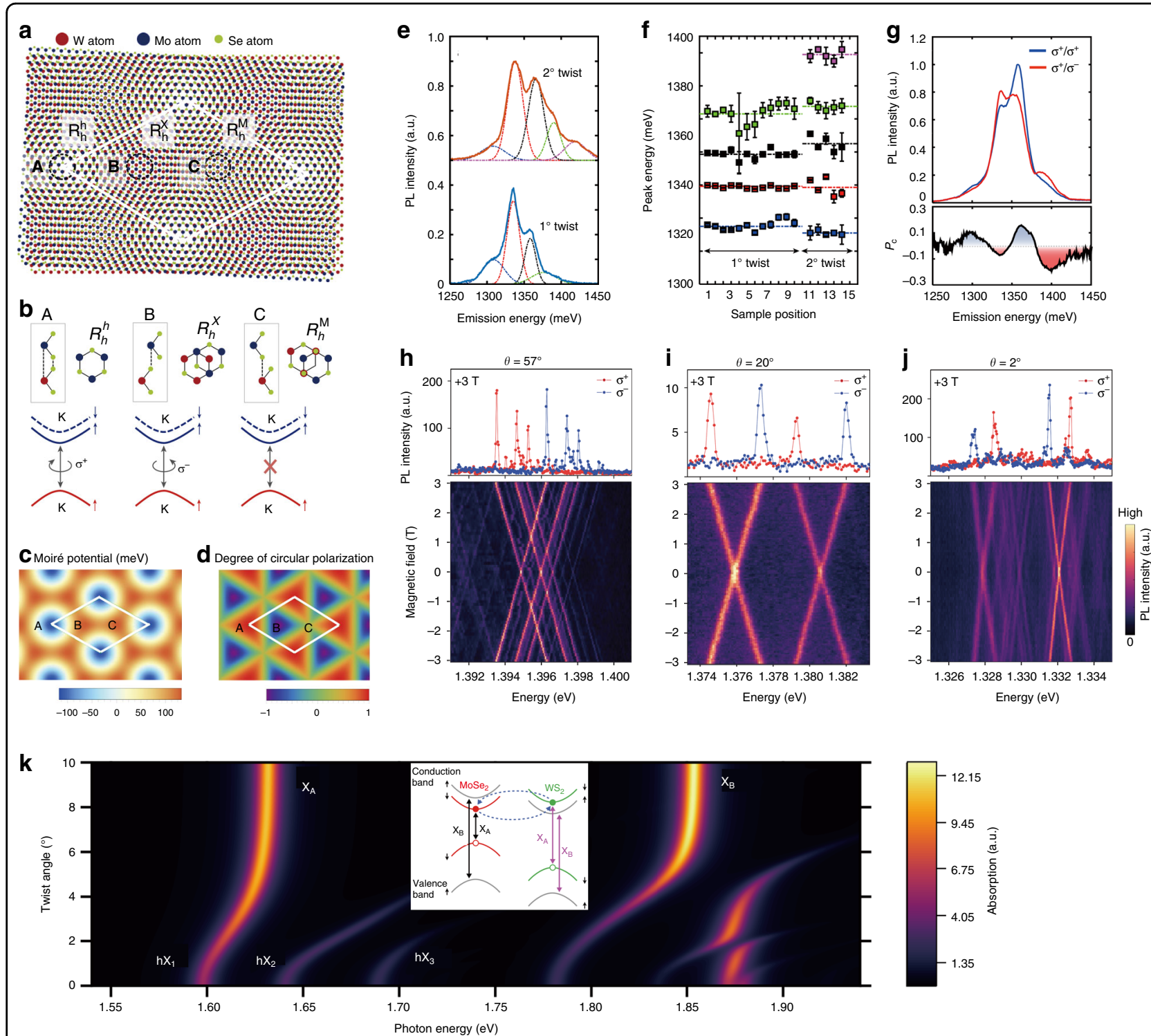

Fig. 4 Moiré interlayer exciton. a Moiré pattern in an R-type $\mathrm{MoSe}_{2} \mathrm{WSe}_{2}$ heterobilayer ${ }^{150}$. The three highlighted regions (A, B, and C sites) correspond to the local atomic configurations with three-fold rotational symmetry. $\mathbf{b}$ Side-views and top-views of the three R-type local atomic registries $\left(A, B\right.$, and $C$ sites) and the corresponding optical selection rules for interlayer excitons in these atomic registries ${ }^{150}$. The interlayer exciton emission at the A (B) site is left-circularly (right-circularly) polarized, while that at the $C$ site is transition-forbidden under normal incidence. c Moiré potential of the interlayer exciton transition with a local minimum at site $A^{150}$. d Optical selection rules for K-valley interlayer excitons ${ }^{150}$. The high-symmetry $A$ and $B$ sites are circularly polarized with opposite signs, and the regions in between are elliptically polarized. e PL spectra of multiple moiré interlayer excitons in $\mathrm{MoSe}_{2} / \mathrm{WSe}_{2}$ heterobilayers with twist angles of $1^{\circ}$ (bottom) and $2^{\circ}$ (top). Each spectrum is fitted with four $\left(1^{\circ}\right)$ or five $\left(2^{\circ}\right)$ Gaussian functions ${ }^{150}$. f The center energy of each moiré interlayer exciton resonance at different spatial positions across each sample ${ }^{150}$. The average peak spacing for a twist angle of $1^{\circ}\left(2^{\circ}\right)$ is $22 \pm 2 \mathrm{meV}(27 \pm 3 \mathrm{meV})$. $g$ Circularly polarized PL spectrum of the $1^{\circ}$ sample under $\sigma+$ excitation (top) $)^{150}$. The degree of circular polarization versus the emission wavelength is shown at the bottom, demonstrating multiple moiré interlayer excitons with alternating co-circularly and crosscircularly polarized emissions. $\mathbf{h}-\mathbf{j}$ Magnetic-field-dependent PL from moiré-trapped interlayer excitons in $\mathrm{MoSe}_{2} / \mathrm{WSe}_{2}$ heterobilayers with twist angles of $57^{\circ}(\mathbf{h}), 20^{\circ} \mathbf{( i )}$, and $\left.2^{\circ} \mathbf{( j )}\right)^{141}$. Top: Circular polarization-resolved PL spectra with narrow linewidth $(100 \mu \mathrm{eV})$ at $3 \mathrm{~T}$. The excitation is linearly polarized, and the $\sigma^{+}$and $\sigma^{-}$components of the PL emission are shown in red and blue, respectively. Bottom: total PL intensity as a function of magnetic field, displaying a linear Zeeman shift of the $\sigma^{+}$-polarized and $\sigma^{-}$-polarized components. The derived effective $g$-factors from Zeeman splitting are $-15.89 \pm$ $0.02,-15.79 \pm 0.05$, and $6.72 \pm 0.02$ for samples with twist angles of $57^{\circ}, 20^{\circ}$, and $2^{\circ}$, respectively. $\mathbf{k}$ Absorption spectrum of the MoSe $2 \mathrm{WS}_{2}$ heterobilayer as a function of the twist angle ${ }^{155}$. The $\mathrm{MoSe}_{2} \mathrm{~A}$-exciton and B-exciton resonances $\left(X_{\mathrm{A}}\right.$ and $\left.X_{\mathrm{B}}\right)$ are indicated for large twist angles where hybridization effects become negligible. The three resonances labeled $h X_{1,2,3}$ appearing at $\theta \approx 0^{\circ}$ correspond to the hybridized excitons in the vicinity of $X_{A}$. Those in the vicinity of $X_{B}$ are not labeled. Specifically, $h X_{3}$ results from the hybridization of the first folding of the $X_{A}$ band into the mini Brillouin zone (the reduced BZ of the moiré superlattice), a direct signature of the moiré superlattice effect. a- $\mathbf{g}$ Reprinted with permission from ref. ${ }^{150}$ [Springer Nature Limited]. h-j Reprinted with permission from ref. ${ }^{141}$ [Springer Nature Limited]. k Reprinted with permission from ref. ${ }^{155}$ [Springer Nature Limited] 
confining/trapping excitons with a potential modulation depth of $\sim 100-250 \mathrm{meV}$, and the local potential minima sitting at those high-symmetry sites within the superlattice (Fig. 4c) ${ }^{18,36,148-151}$.

Obviously, for interlayer excitons with electrons and holes separated in opposite layers, their properties should be substantially affected by the interlayer configurations and thus by the moiré pattern, which carries periodically varied interlayer atomic registries. As proposed, an intuitive impact of the moiré pattern on interlayer excitons is that it is able to trap them in the minima of the moiré potential, and thereby, an ordered nanoscale quantum emitter array or an excitonic superlattice could be realized with interlayer excitons by designing an appropriate moiré period and/or the profile of the moiré potential, which can be tuned via an external electric field or strain ${ }^{36,69}$. Another intriguing effect from the moiré pattern is the lateral modulation of the optical selection rules within the superlattice ${ }^{36,150}$. That is, an interlayer exciton located at the highsymmetry site $R_{\mathrm{h}}^{\mathrm{h}}\left(R_{\mathrm{h}}^{\mathrm{X}}\right)$ couples only to $\sigma^{+}\left(\sigma^{-}\right)$circularly polarized light for a specific valley and at sites in between is elliptically polarized, while an interlayer exciton located at site $R_{\mathrm{h}}^{\mathrm{M}}$ couples to out-of-plane polarization light (Fig. 4b, d) ${ }^{150}$. This means that even interlayer excitons in the same spin-valley configuration can couple to polarized light with opposite helicities due to the preserved $\hat{C}_{3}$ symmetry at these high-symmetry sites, suggesting that circular polarization selection rules are no longer locked to the valley index. In other words, the optical selection rules in such heterobilayers are determined not only by the atomic quantum number (associated with the spin, orbital, and valley quasiparticle angular momenta (QAM)), but also by the interlayer translation that characterizes the local interlayer atomic registry in the moiré superlattice (referring to moiré QAM) ${ }^{152}$. Therefore, a new degree of freedom, i.e., the moiré degree of freedom, is introduced to better characterize and manipulate the physical properties of interlayer excitons in the moiré pattern. The optical dipole oscillator strength and radiative lifetime of interlayer excitons were reported to be modulated on a few orders of magnitude across the superlattice by such a moiré effect ${ }^{148}$. Moreover, the emerging periodicity from the strong-coupling moiré superlattices could result in folding of the interlayer (intralayer) exciton bands into a mini-Brillouin zone (a reduced BZ of the moiré superlattice), forming moiré exciton minibands manifested as multiple exciton resonances with different optical selection rules in the optical spectra ${ }^{36,41,116,146}$.

Note that the discussed intriguing phenomena realized by the moiré effect have so far been explored mainly by theoretical calculations in twisted TMD vdW heterobilayers. Recently, multiple moiré interlayer exciton resonances with alternating co-circularly and crosscircularly polarized emission and almost a constant peak spacing ( 22-27 meV) were experimentally observed in a $\mathrm{MoSe}_{2} / \mathrm{WSe}_{2}$ heterobilayer by far-field optical measurements at $15 \mathrm{~K}$ (Fig. $4 \mathrm{e}-\mathrm{g}$ ) ${ }^{150}$. These resonances were attributed to the excitonic ground and excited states confined within the moiré potential with a depth of $\sim 100 \mathrm{meV}$, consistent with theoretical calculations. Subsequently, multiple interlayer exciton states with opposite optical selection rules were reported in a $\mathrm{WSe}_{2} / \mathrm{WS}_{2}$ moiré superlattice, and their spin, valley and moiré QAM, which contribute to the optical selection rules, were unambiguously determined via novel resonant optical pump-probe spectroscopy and PL excitation spectroscopy ${ }^{152}$. Moiré-trapped interlayer excitons with narrow linewidth $(100 \mu \mathrm{eV})$, circularly polarized optical selection rules and large Landé $g$-factor at various twist angles were also evidenced in $\mathrm{MoSe}_{2} / \mathrm{WSe}_{2}$ heterobilayers by both optical and magneto-PL spectroscopy under very low excitation power $(20 \mathrm{nW})$ and temperature $(1.6 \mathrm{~K})(\text { Fig. } 4 \mathrm{~h}-\mathrm{j})^{141}$. Such moiré-trapped interlayer excitons with characterized narrow linewidths were recently reported in a trilayer heterostructure with a $\mathrm{WSe}_{2}$ monolayer below the $\mathrm{MoSe}_{2}$ bilayer, where the resulting two quantum-confined interlayer excitons possessed distinct spin-layer-valley configurations (with parallel and antiparallel spin-valley-locked magnetic moments) ${ }^{153}$. Although intralayer excitons are less influenced by the moiré effect, when the moiré potential is sufficiently strong, such as up to $\sim 250 \mathrm{meV}$, multiple moiré intralayer exciton resonances can be experimentally detected, as revealed in $\mathrm{WSe}_{2} / \mathrm{WS}_{2}$ heterostructure superlattices ${ }^{151}$. More intriguingly, hybridization of the moiré intra- and interlayer exciton minibands could even occur in the moiré mini-Brillouin zone when the $\mathrm{CB}$ edges of the constituent monolayers are closely aligned, leading to hybridized excitons that inherit both the brightness of intralayer excitons and the polar nature of interlayer excitons, accompanied by a pronounced twist-angle-controlled energy shift (Fig. 4k) ${ }^{155}$. Such a hybridization effect was also experimentally demonstrated in homobilayers, along with the observation of an incompressible Mott-like state of electrons at half-filling of each layer ${ }^{47,154}$. Additionally, hybridization between the moiré interlayer excitons and photons in a planar 2D cavity could be possible and result in two types of moiré polaritons with distinct forms of topological transport phenomena such as spin/valley Hall and polarization Hall effects ${ }^{156}$. In summary, moiré superlattices provide a rich playground for exploring new quantum phenomena in $\mathrm{vdW}$ heterostructures, which undoubtedly will facilitate their application in various exciting areas such as nanophotonics and quantum information processing. 


\section{Interlayer exciton relaxation in TMD vdW heterostructures}

In this section, we review the population recombination dynamics of interlayer excitons, the intervalley scattering process, and the valley polarization dynamics of interlayer excitons in TMD vdW heterostructures.

\section{Recombination dynamics of interlayer excitons}

Owing to the reduced overlap of the electron and hole wavefunctions of the spatially indirect interlayer exciton, its oscillation strength was reported to be several orders of magnitude lower than that of the intralayer exciton, as stated before ${ }^{80,116}$, which indicates a much longer recombination lifetime, as evidenced in both theoretical and experimental studies ${ }^{22-28,33,42,56,111,157-161}$.

Ultrafast optical measurements such as femtosecond pump-probe and time-resolved PL (TRPL) spectroscopy are typical tools for tracing the recombination dynamics of interlayer excitons. An early report demonstrated direct probing of the transient spectrum and dynamics of interlayer excitons in $\mathrm{MoS}_{2} / \mathrm{WS}_{2}$ heterobilayers by an ultrafast pump-probe technique, with its recombination being tuneable from direct $(\sim 40 \mathrm{ps})$ to indirect $(\sim 1.5 \mathrm{~ns})$ dependent on the stacking orientation (Fig. $5 \mathrm{a})^{42}$. With this technique, the long lifetime property of interlayer excitons could be indirectly reflected by the photobleaching signals of the intralayer excitons in heterobilayers being longer than those in monolayers ${ }^{56,159,160}$. Nevertheless, due to the very small oscillation strength imposing difficulty on directly accessing the absorption transition, PL dynamics are most commonly used to directly reflect the interlayer exciton recombination process, with the reported PL lifetimes ranging from several $\mathrm{ns}$ to hundreds of $\mathrm{ns}$ or even $\sim \mu \mathrm{s}^{22-28,33,111,161}$. Additionally, a number of studies have revealed that the recombination lifetimes of interlayer excitons can be extensively tuned by external factors, such as the electric field $^{26,33}$, temperature ${ }^{23,24,28,161}$, twisting angle ${ }^{56}$, interlayer distance ${ }^{160}$, and optical cavity ${ }^{111}$ due to their interlayer configuration (or spatially indirect nature). Note that double interlayer excitons with two distinctly long-lived lifetimes were observed in certain TMD vdW heterostructures, with their assignments as momentum direct and indirect interlayer excitons ${ }^{23}$, spin-singlet and spintriplet interlayer excitons ${ }^{22,135}$, or neutral and charged interlayer excitons ${ }^{161}$. The origin of these two species and their accurate assignments are of fundamental interest for further exploration.

The decay lifetimes of interlayer excitons in various TMD vdW heterostructures are summarized in Table 1. The interlayer exciton lifetime of $\mathrm{MoSe}_{2} / \mathrm{WSe}_{2}$ heterostructures has been most widely studied and generally consists of two lifetime components: a fast component in tens of ns and a slow component in hundreds of ns.
Some results reported only one of these two lifetimes, either in several ns or in hundreds of ns (Table 1). The accurate assignment of these two lifetimes remains ambiguous, although they were previously ascribed to quasi-direct (fast) and indirect (slow) interlayer excitons $^{23}$. Additionally, longer lifetimes were usually observed at lower temperatures, probably due to suppressed non-radiative recombination. Note that a dark exciton in the $\mathrm{MoSe}_{2} / \mathrm{WSe}_{2}$ heterostructure reportedly had a lifetime on a microsecond timescale, and could serve as a microsecond reservoir for an interlayer exci$\operatorname{ton}^{27}$, which is quite promising for the realization of excitonic devices with long exciton transport distances. The interlayer exciton lifetime in $\mathrm{WS}_{2} / \mathrm{WSe}_{2}$ heterostructures was reported to be relatively short, with a value within $1 \mathrm{~ns}$, which could be elongated to several ns or longer than 10 ns by either lowering the temperature or increasing the interlayer distance between the constituent monolayers by inserting hBN layers (Table 1). The $\mathrm{MoS}_{2} /$ $\mathrm{WS}_{2}$ heterostructure was also reported to have a relatively short interlayer exciton lifetime, with values on the order of tens of ps or on the order of ns depending on the stacking mode between layers ${ }^{42}$. Such a stacking mode or twist angle dependence was also revealed in the $\mathrm{MoS}_{2}$ / $\mathrm{WSe}_{2}$ heterostructure, with lifetimes ranging from $\sim 50 \mathrm{ps}$ to $\sim 3 \mathrm{~ns}^{56}$. Surprisingly, the interlayer exciton lifetime in the hBN-capsulated $\mathrm{MoS}_{2} / \mathrm{WS}_{2}$ heterostructure was found to be significantly longer $(\sim 100 \mathrm{~ns})^{26}$ than that of the above uncapsulated $\mathrm{MoS}_{2} / \mathrm{WS}_{2}$ heterostructure ${ }^{42}$, the reason for which is obscure and may be related to complicated factors such as the different material structures, fabrication methods, temperatures, twist angles between layers, and detection techniques.

Additionally, the interlayer exciton lifetimes obtained by the pump-probe technique were generally shorter than those detected by TRPL spectroscopy for the heterobilayers (Table 1). Due to the small oscillation strength of the interlayer excitons, the pump-probe technique normally detected the electron/hole dynamics of the constituent monolayers to reflect the interlayer exciton lifetime, while TRPL directly detected the PL dynamics of the interlayer excitons. In addition, the pump-probe method was generally performed at room temperature or at higher temperatures in the currently reported works (for those specified in the works). Both of these factors may affect the detected interlayer exciton lifetimes. Nevertheless, the clear origin of these discrepancies needs further investigation. Interestingly, interlayer excitons were also found in vdW heterostructures with three constituent monolayers. For instance, a long-lived interlayer exciton emission was reported in the $\mathrm{MoS}_{2} / \mathrm{MoSe}_{2} /$ $\mathrm{MoS}_{2}$ heterostructure with two lifetime components ( $\sim 5$ and $\sim 135 \mathrm{~ns}$, ascribed to momentum-direct and momentum-indirect interlayer excitons $)^{24}$. In another 


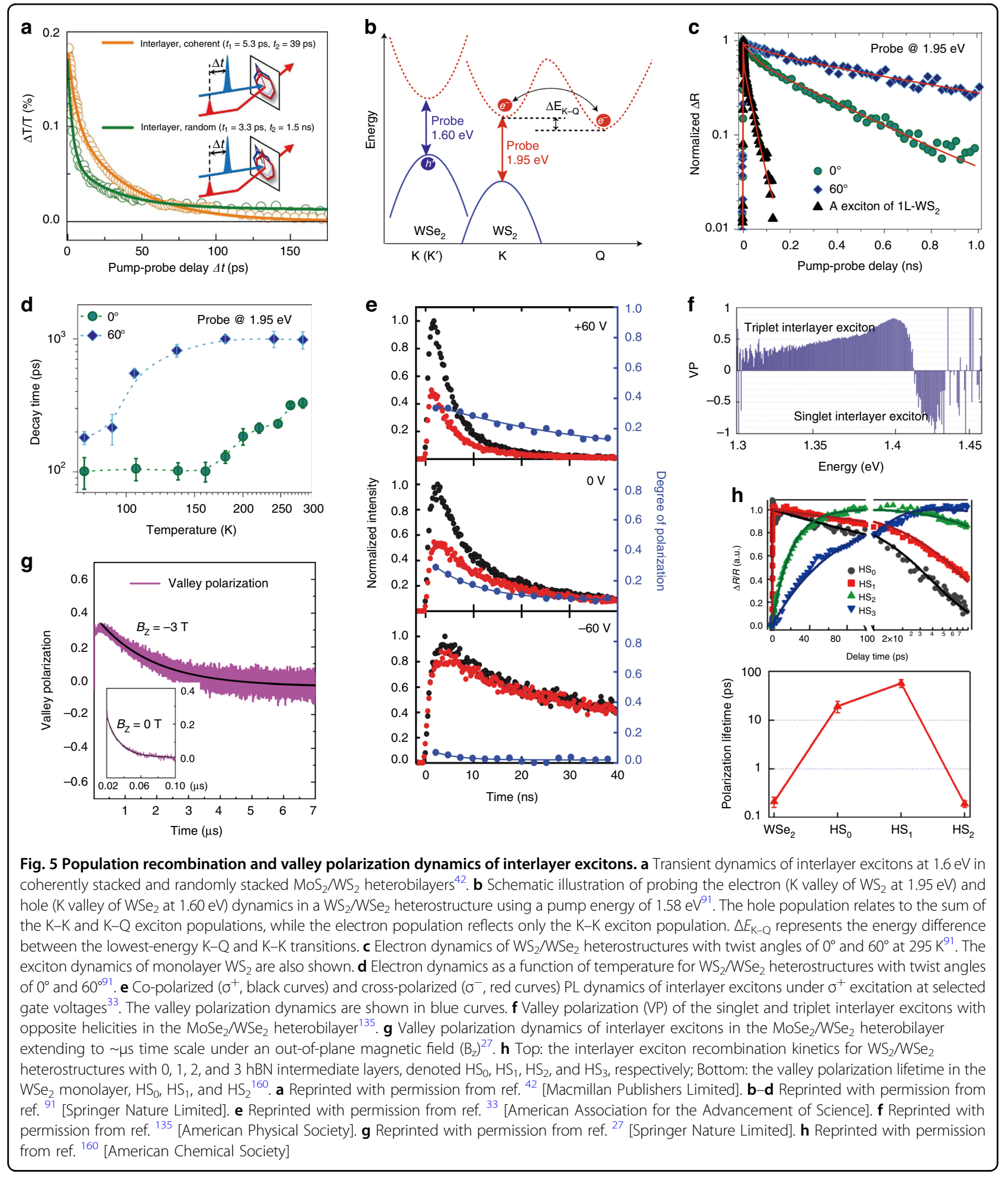

trilayer heterostructure, $\mathrm{WSe}_{2} / \mathrm{MoSe}_{2} / \mathrm{WSe}_{2}{ }^{161}$, both neutral and charged interlayer excitons were spectrally resolved with lifetimes on the order of ns, which decreased linearly as the temperature increased, most likely due to enhanced non-radiative recombination at higher temperatures. Compared to the bilayer $\mathrm{MoSe}_{2} /$ $\mathrm{WSe}_{2}$, the interlayer excitons formed in the trilayer $\mathrm{WSe}_{2} /$ $\mathrm{MoSe}_{2} / \mathrm{WSe}_{2}$ were demonstrated to have greater electronhole wavefunction overlap and thus stronger oscillation strength, which may account for the observed lifetime 
Table 1 Decay lifetimes of interlayer excitons in various TMD vdW heterostructures

\begin{tabular}{|c|c|c|c|c|c|c|c|c|c|}
\hline \multirow[t]{2}{*}{ HSs } & \multirow[t]{2}{*}{ Fabrication method } & \multirow[t]{2}{*}{ Twist angle $\left({ }^{\circ}\right)$} & \multicolumn{3}{|c|}{ Lifetime (ns) } & \multirow[t]{2}{*}{ Temp (K) } & \multirow[t]{2}{*}{ Technique } & \multirow[t]{2}{*}{ Notes } & \multirow[t]{2}{*}{ Refs } \\
\hline & & & $\tau_{1}$ & $\tau_{2}$ & $\tau_{3}$ & & & & \\
\hline \multirow[t]{15}{*}{$\mathrm{MoSe}_{2} / \mathrm{WSe}_{2}$} & MEwet transfer & & 1.80 & & & 20 & TRPL & & 22 \\
\hline & MEdry transfer & 0 & $\sim 10$ & & & 30 & TRPL & & 33 \\
\hline & MEdry transfer & & 16 & 138 & & 4.5 & TRPL & & 28 \\
\hline & MEdry transfer & 54 & 40 & $>100$ & & 4 & TRPL & $B=0 T$ & 25 \\
\hline & & 54 & 70 & $>200$ & & 4 & TRPL & $B=28 \mathrm{~T}$ & \\
\hline & MEdry transfer & $0 / 60$ & 10 & 100 & & 4 & TRPL & $E_{\mathrm{HS}}=0 \mathrm{~V} / \mathrm{nm}$ & 30 \\
\hline & & $0 / 60$ & & 600 & & 4 & TRPL & $E_{\mathrm{HS}}>0.1 \mathrm{~V} / \mathrm{nm}$ & \\
\hline & MEdry transfer & 60 & $\sim 50$ & $\sim 100$ & & 3 & TRPL & & 23 \\
\hline & MEdry transfer & $0 / 60$ & & $\sim 1000$ & & & TRPL & Dark exciton & 27 \\
\hline & MEdry transfer & 58.7 & 2.30 & & & & TRPL & & 135 \\
\hline & MEdry transfer & 60 & $\sim 12$ & & & 4 & TRPL & Moiré interlayer exciton & 142 \\
\hline & MEdry transfer & 3.5 & & 100 & & & TRPL & Moiré period (5 nm) & 162 \\
\hline & MEdry transfer & 1.1 & 1.00 & & & & TRPL & Moiré period (17 nm) & \\
\hline & CVD & 0 & 1.20 & 5.50 & & & TRPL & No moiré pattern & \\
\hline & $C V D$ & 60 & 6.00 & 44 & 877 & 4.2 & TRPL & No moiré pattern & 111 \\
\hline \multirow[t]{2}{*}{$\mathrm{WS}_{2} / \mathrm{WSe}_{2}$} & $C V D$ & 0,60 & 0.97 & & & 295 & Pump-probe & Hole dynamics $\left(\mathrm{WSe}_{2}\right)$ & 91 \\
\hline & & 0,60 & 4.10 & & & 78 & Pump-probe & Hole dynamics $\left(\mathrm{WSe}_{2}\right)$ & \\
\hline \multirow[t]{2}{*}{$\mathrm{WS}_{2} / \mathrm{WSe}_{2}$} & CVD & 0 & 0.36 & & & 295 & Pump-probe & Electron dynamics $\left(\mathrm{WS}_{2}\right)$ & 91 \\
\hline & & 60 & 0.98 & & & 295 & Pump-probe & Electron dynamics $\left(\mathrm{WS}_{2}\right)$ & \\
\hline$W_{2} / \mathrm{WSe}_{2}$ & MEdry transfer & & 0.41 & & & & Pump-probe & Electron dynamics $\left(\mathrm{WS}_{2}\right)$ & 160 \\
\hline $\mathrm{WS}_{2} / \mathrm{hBN} / \mathrm{WSe}_{2}$ & MEdry transfer & & 1.00 & & & & Pump-probe & Electron dynamics $\left(\mathrm{WS}_{2}\right)$ & 160 \\
\hline WS $_{2} /$ bilayer-hBN/WSe ${ }_{2}$ & MEdry transfer & & 4.96 & & & & Pump-probe & Electron dynamics $\left(\mathrm{WS}_{2}\right)$ & 160 \\
\hline $\mathrm{WS}_{2} /$ trilayer-hBN/WSe${ }_{2}$ & MEdry transfer & & $>10$ & & & & Pump-probe & Electron dynamics $\left(\mathrm{WS}_{2}\right)$ & 160 \\
\hline \multirow[t]{2}{*}{$\mathrm{MoS}_{2} \mathrm{WS}_{2}$} & $\begin{array}{l}\text { Vertical } \\
\text { heteroepitaxial growth }\end{array}$ & Coherent stack & 0.04 & & & & Pump-probe & & 42 \\
\hline & Manual stacking & Random stack & 1.50 & & & & Pump-probe & & \\
\hline \multirow[t]{2}{*}{$\mathrm{hBN} / \mathrm{MoS}_{2} / \mathrm{WS}_{2} / \mathrm{hBN}$} & MEdry transfer & $0 / 60$ & & $\sim 100$ & & 10 & TRPL & $V_{G}=0-4.5 \mathrm{~V}$ & 26 \\
\hline & & & & $\sim 400$ & & 10 & TRPL & $V_{G}=-4.5 \mathrm{~V}$ & \\
\hline $\mathrm{MoS}_{2} / \mathrm{WSe}_{2}$ & MEdry/wet transfer & & $\begin{array}{l}\sim 0.05 \text { to } \\
\sim 3\end{array}$ & & & & Pump-probe & $\begin{array}{l}\text { Depending on the } \\
\text { twist angle }\end{array}$ & 56 \\
\hline $\mathrm{MoS}_{2} / \mathrm{MoSe}_{2} / \mathrm{MoS}_{2}$ & CVDwet transfer & & 5 & 135 & & 6 & TRPL & & 24 \\
\hline \multirow[t]{4}{*}{$\mathrm{WSe}_{2} / \mathrm{MoSe}_{2} / \mathrm{WSe}_{2}$} & MEdry transfer & & 2.54 & & & 4 & TRPL & Neutral interlayer exciton & 161 \\
\hline & & & 2.47 & & & 40 & & & \\
\hline & & & 1.84 & & & 80 & & & \\
\hline & & & 0.41 & & & 120 & & & \\
\hline \multirow[t]{2}{*}{$\mathrm{WSe}_{2} / \mathrm{MoSe}_{2} / \mathrm{WSe}_{2}$} & MEdry transfer & & 1.24 & & & 4 & TRPL & Charged interlayer exciton & 161 \\
\hline & & & 0.47 & & & 20 & & & \\
\hline
\end{tabular}

HSs heterostructures, Temp temperature, ME mechanical exfoliation, CVD chemical vapor deposition, TRPL time-resolved photoluminescence spectroscopy, $B$ applied out-of-plane magnetic field, $E_{\mathrm{HS}}$ electric field across the heterostructure, $V G$ externally applied voltage, Ref reference

discrepancies between these two types of heterostructures. Lifetime modulation of the interlayer exciton by changing its electron-hole wavefunction overlap was also realized by applying an external electric field ${ }^{26,30}$, or by tuning the interlayer distance as described above ${ }^{160}$, with a longer interlayer exciton lifetime observed with reducing electron-hole wavefunction overlap.

Moreover, the role of the moire superlattice on the interlayer exciton lifetime was discussed recently. A twist angle dependence of the interlayer exciton lifetime was demonstrated in the $\mathrm{WS}_{2} / \mathrm{WSe}_{2}$ heterostructure, which exhibited a deeper moiré potential for a twist angle of $0^{\circ}$ than a twist angle of $60^{\circ}$ (Fig. $\left.5 \mathrm{~b}-\mathrm{d}\right)^{91}$. Figure $5 \mathrm{~b}$ shows a schematic illustration of probing the electron and hole dynamics in the $\mathrm{WS}_{2} / \mathrm{WSe}_{2}$ heterostructure to reflect the interlayer exciton dynamics by using the pump-probe technique with a pump energy of $1.58 \mathrm{eV}$. As stated in this work, the hole population (in the $\mathrm{K}$ valley of $\mathrm{WSe}_{2}$ at $1.60 \mathrm{eV}$ ) monitored the sum of the $\mathrm{K}-\mathrm{K}$ and $\mathrm{K}-\mathrm{Q}$ interlayer exciton populations, while the electron population (in the $\mathrm{K}$ valley of $\mathrm{WS}_{2}$ at $1.95 \mathrm{eV}$ ) reflected only the $\mathrm{K}-\mathrm{K}$ exciton population. As shown in Fig. 5c, the dynamics probed at $1.95 \mathrm{eV}$ (related to the $\mathrm{K}-\mathrm{K}$ interlayer exciton) exhibited a substantial twist angle dependence, with the decay lifetime for $60^{\circ}$ being three times longer than that for $0^{\circ}$ at $295 \mathrm{~K}$. Both lifetimes decreased as the temperature 
decreased (Fig. 5d). Such twist angle-dependent and temperature-dependent dynamics were explained by the intraband scattering of the electrons between the $\mathrm{K}$ and $\mathrm{Q}$ valleys. Since the moiré potential for $0^{\circ}$ was deeper or larger than that for $60^{\circ}$, as was the energy difference between the lowest-energy $\mathrm{K}-\mathrm{Q}$ and $\mathrm{K}-\mathrm{K}$ transitions $\left(\Delta E_{\mathrm{K}-\mathrm{Q}}\right)^{91}$, the larger $\Delta E_{\mathrm{K}-\mathrm{Q}}$ for $0^{\circ}$ resulted in a less efficient backscattering of electrons from $\mathrm{Q}$ to $\mathrm{K}$ and thus a shorter $\mathrm{K}-\mathrm{K}$ exciton decay lifetime than those for $60^{\circ}$. This was consistent with the phenomenon of the temperature dependence of the lifetimes. At higher temperatures, more electrons could be back-scattered from the $\mathrm{Q}$ to $\mathrm{K}$ valley by phonon absorption, which could increase the $\mathrm{K}-\mathrm{K}$ exciton population and hence give rise to a longer decay lifetime (Fig. 5d). Note that a higher temperature was required to back-scatter electrons for $0^{\circ}$ than for $60^{\circ}$ due to the larger $\Delta E_{\mathrm{K}-\mathrm{Q}}$ for $0^{\circ}$ (Fig. $5 \mathrm{~d}$ ). The moiré effect on the interlayer exciton lifetime was also studied in $\mathrm{MoSe}_{2} / \mathrm{WSe}_{2}$ heterostructures prepared by chemical vapor deposition (CVD, twist angle of $0^{\circ}$ ) and mechanical stacking with twist angles of $\sim 1.1^{\circ}$ and $\sim 3.5^{\circ 162}$. The sample at a twist angle of $0^{\circ}$ without a moiré pattern possessed an averaged interlayer exciton lifetime of $\sim 1.7 \mathrm{~ns}$, which was similar to that at a twist angle of $\sim 1.1^{\circ}$ with a moiré period of $\sim 17 \mathrm{~nm}$. However, the interlayer exciton lifetime in the sample with a twist angle of $\sim 3.5^{\circ}$ and a moiré period of $\sim 5 \mathrm{~nm}$ was significantly longer $(\sim 100 \mathrm{~ns})$, which was ascribed to the larger momentum mismatch between the CBM and VBM at this twist angle, thus inducing an indirect transition in both real and momentum space.

To date, the interlayer exciton lifetimes studied in various TMD vdW heterostructures have been discussed, with the reported values covering a broad range from nanoseconds to microseconds. Many factors can influence the interlayer exciton lifetime such as the temperature, the degree of electron-hole overlap, and the moiré superlattice. Generally, longer lifetimes were observed at lower temperatures, probably due to suppressed nonradiative recombination, except for the case with the moiré effect ${ }^{91}$. The electron-hole wavefunction overlap of the interlayer exciton should be the main source for modulating its lifetime, and it could be influenced by various factors such as the material structure, fabrication method, twist angle, and external electric field, giving rise to a longer lifetime under a smaller electron-hole overlap. Although more exploration is needed to clarify the discrepancies in these studies, the observed longer recombination lifetimes of interlayer excitons compared to those of intralayer excitons, plus the existing repulsive interactions, make interlayer excitons quite suitable for exploitation in excitonic devices with long-distance exciton transport and promising for macroscopic quantum state generation for the realization of Bose-Einstein condensation and superfluidity.

\section{Intervalley scattering process}

Intervalley scattering plays a key role in determining the valley polarization lifetimes of materials for valleytronic applications. Due to the valley-dependent optical selection rules and the spin-valley locking effect, the intervalley scattering in TMD monolayers was expected to be slow due to the large momentum mismatch along with a simultaneous spin flip between the $+\mathrm{K}$ and $-\mathrm{K}$ valleys ${ }^{3,133}$. However, this was in contrast to the experimental observations, with the reported spin-valley lifetimes on a time scale of a few picoseconds in TMD monolayers even at low temperatures ${ }^{163-166}$. Such contradictory findings were attributed to the strong electronhole exchange interactions (via the Maialle-Silva-Sham mechanism) for the intralayer excitons in monolayers ${ }^{167-169}$, which significantly strengthen the intervalley scattering process and thus shorten the spin-valley lifetimes. Undoubtedly, this will impede the application of TMDs in spintronics/valleytronics if intralayer excitons are used as spin-valley information carriers.

On the other hand, the interlayer exciton with transferred spin-valley polarization has been considered a good candidate for exploring valleytronics. Since the electron-hole exchange strength is proportional to the oscillation strength ${ }^{168}$, such an exchange interaction could be dramatically weakened for the interlayer exciton due to its oscillation strength being much smaller than that of the intralayer exciton. Therefore, the intervalley scattering process of the interlayer exciton should be strongly suppressed ${ }^{167}$, giving rise to significantly extended valley lifetimes, as discussed below.

\section{Valley polarization dynamics of interlayer excitons}

Owing to their intrinsic valley-contrasting physics and spin-valley coupling properties ${ }^{132,133}$, TMDs offer an appealing platform for developing spintronic/valleytronic devices by using spin-valley pseudospin, which can potentially circumvent the limitations of speed and power consumption imposed by electron charges. A sufficiently long spin-valley lifetime is essential for such applications to maintain the spin-valley information for subsequent signal processing or communication.

Interlayer excitons, which have a long population lifetime and suppressed intervalley scattering, as discussed above, have been considered promising spin-valley information carriers due to their valley polarization lifetime being longer than that of intralayer excitons. An early study demonstrated $\sim 30 \%$ valley-polarized interlayer exciton emission at $30 \mathrm{~K}$ in a $\mathrm{MoSe}_{2} / \mathrm{WSe}_{2}$ heterobilayer, with a remarkably enhanced valley polarization lifetime of a few nanoseconds (compared to the few picosecond valley lifetime of intralayer excitons $)^{33}$. The degree of valley polarization could be further electrically controlled by the gate, with reported valley polarization lifetimes of $\sim 39$, 
$\sim 10$, and $\sim 5 \mathrm{~ns}$ for gate voltages of $+60,0$, and $-60 \mathrm{~V}$, respectively (Fig. 5e) ${ }^{33}$. This phenomenon was most likely due to the larger interlayer separation of the electron and hole at positive gate voltages; thus, a smaller oscillation strength and weaker intervalley scattering resulted in a higher valley polarization and longer valley polarization lifetime. However, obtaining a solid microscopic mechanism responsible for the observed gate-dependent valley polarization lifetimes requires thorough future investigations. A much higher degree of valley polarization approaching unity or over $80 \%$ with a valley polarization lifetime on tens of nanoseconds was later achieved for both singlet and triplet interlayer excitons in $\mathrm{MoSe}_{2} / \mathrm{WSe}_{2}$ heterobilayers encapsulated with hexagon-boron nitride $(\mathrm{hBN})$ layers at cryogenic temperatures (Fig. $5 \mathrm{f})^{135}$. This high valley polarization was generally ascribed to the ultrafast spin-valley conserved charge transfer as well as the suppressed intervalley scattering of the interlayer excitons. Alternatively, valley polarization could be enhanced by applying an out-of-plane magnetic field $\left(\mathbf{B}_{\mathrm{z}}\right)$ to lift the valley degeneracy via the valley-Zeeman effect $^{25,27,110}$. Giant valley-Zeeman splitting $(\sim 26 \mathrm{meV})$ of the interlayer excitons with near-unity valley polarization and a large effective $g$-factor $(-15)$ was observed in ABstacked (stacking angle close to $60^{\circ}$ ) $\mathrm{MoSe}_{2} / \mathrm{WSe}_{2}$ heterobilayers under a $30 \mathrm{~T}$ magnetic field ${ }^{25}$. As expected, the valley polarization lifetime was extended under the magnetic field, with the reported value increasing from $\sim 15 \mathrm{~ns}$ $\left(\mathbf{B}_{\mathrm{z}}=0 \mathrm{~T} \text { ) to } \sim 1.75 \mu \mathrm{s} \text { at } \mathbf{B}_{\mathrm{z}}=-3 \mathrm{~T} \text { (Fig. } 5 \mathrm{~g}\right)^{27}$. In addition to the electric and magnetic field control of the valley polarization and its dynamics, structural tuning by inserting $\mathrm{hBN}$ intermediate layers between the bilayer to modulate the interlayer electron-hole Coulomb interaction was recently demonstrated to precisely control the interlayer exciton recombination and the valley polarization dynamics (Fig. 5h) ${ }^{160}$. Notably, a single particle such as the resident hole in the heterobilayer emerged as a good valley information carrier. This single particle could have a much longer valley polarization lifetime $\left(>20^{170}\right.$ or $40 \mu \mathrm{s}^{171}$ at low temperature) than the interlayer exciton owing to the lack of electron-hole exchange interaction and the large momentum mismatch, and the spin-orbit coupling resulted in a high energy barrier in the VBs between the $K$ valleys, all of which could dramatically suppress the intervalley scattering and prolong the recombination lifetime to substantially extend the valley polarization lifetime.

According to the reported results, it is obvious that the valley polarization and the dynamics of the interlayer exciton basically rely on three factors: (1) the initial degree of valley polarization inherited from the intralayer exciton, (2) the interlayer exciton recombination lifetime, and (3) the intervalley scattering of the interlayer exciton. The first factor is tightly linked to the interlayer spin-valley conserved charge transfer and the intralayer recombination, as well as the intralayer valley depolarization process. Higher valley polarization inherited by the interlayer exciton is expected if interlayer charge transfer occurs at timescales much faster than both intralayer recombination and valley depolarization. The second influencing factor is straightforward since no valley-polarized interlayer excitons exist when their population decays to zero. The final factor is closely related to the electron-hole exchange interaction, as stated above. All these factors ought to be strongly affected by the interlayer coupling in the heterostructures, and additional efforts to engineer these factors to further extend the valley polarization lifetimes of the interlayer excitons are expected.

\section{Interlayer exciton transport in TMD vdW heterostructures}

In this section, we review the interlayer exciton diffusion and drift, valley-polarized interlayer exciton transport, and interlayer exciton transport under moiré potential in TMD vdW heterostructures.

\section{Interlayer exciton diffusion and drift}

Solid-state devices based on the manipulation of excitons hold great potential for bridging the optical communication and signal processing modules in integrated circuits $^{172,173}$. Controllable manipulation of the exciton transport in these devices requires that excitons possess a sufficiently long lifetime to travel over large distances, and that their energy is sensitive to the applied electric field ${ }^{172,173}$. Apparently, the conventional exciton in directgap semiconductors has difficulty meeting such requirements due to its short recombination lifetime $(<1 \mathrm{~ns})$ and the absence of a built-in dipole moment ${ }^{174,175}$. However, a spatially indirect exciton, with a permanent dipole moment and a lifetime several orders of magnitude longer than that of conventional excitons, is a good candidate for these excitonic devices. In fact, indirect excitons formed in traditional semiconductor coupled quantum wells (CQWs) have been extensively explored, with observed lifetimes varying from nanoseconds to microseconds and transport distances over tens and hundreds of micrometers within the lifetimes ${ }^{176-185}$. On the other hand, the emerged TMD vdW heterostructures provide a new platform for generating indirect excitons with long recombination lifetimes, as discussed above, which has recently ignited intensive interest in studying exciton transport in these systems for potential novel excitonic devices.

For instance, interlayer exciton diffusion in various TMD vdW heterostructures (i.e., a $\mathrm{MoSe}_{2} / \mathrm{hBN} / \mathrm{WSe}_{2}$ heterotrilayer and $\mathrm{MoSe}_{2} / \mathrm{WSe}_{2}$ heterobilayer at cryogenic temperature $(4 \mathrm{~K}))$ were investigated recently ${ }^{30,34}$. Both the PL emission energy and the diffusion distance increased as the laser power increased (Fig. $6 \mathrm{a}-\mathrm{c})^{30}$, which was principally ascribed to density-dependent exciton-exciton 

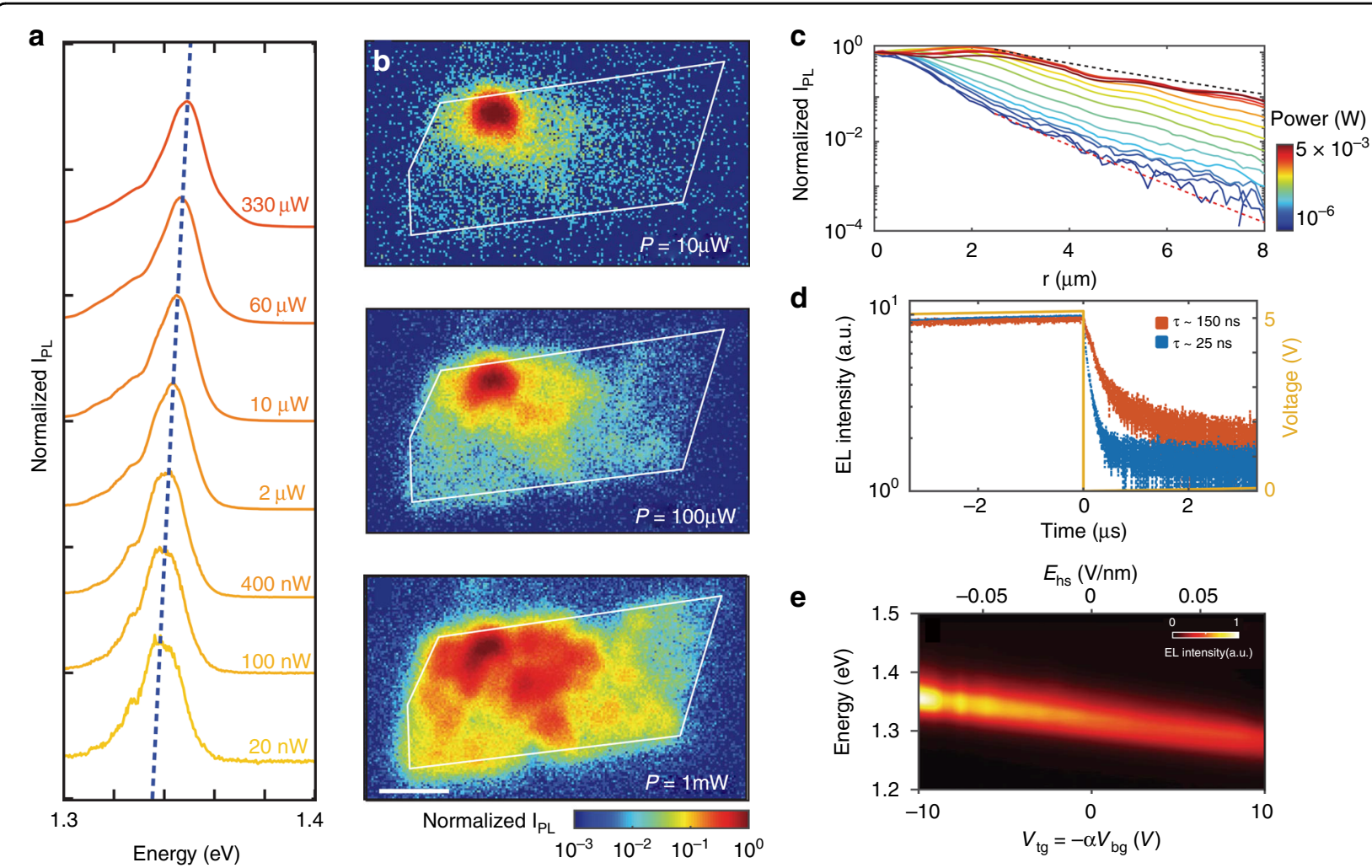

d
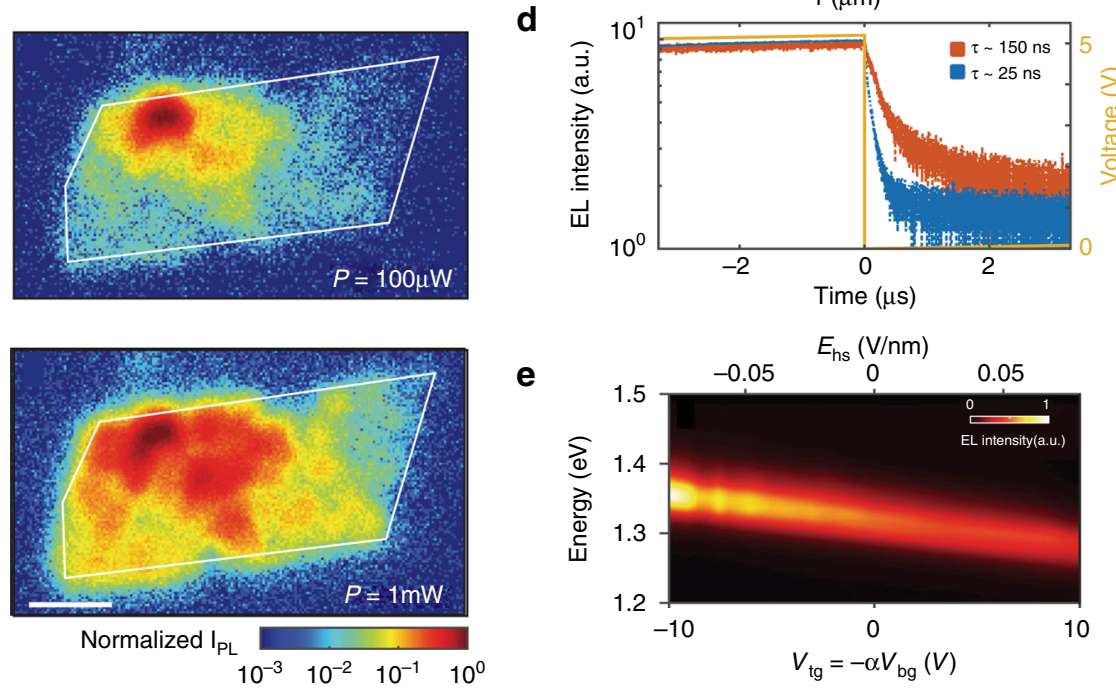

Fig. 6 Interlayer exciton diffusion. a Power dependence of the normalized PL spectra of interlayer excitons in a MoSe $2 / \mathrm{WSe}_{2} \mathrm{heterobilayer}^{30}$. b Spatial dependence of the normalized PL intensity of interlayer excitons for different incident powers (10, 100, and $1000 \mu \mathrm{W})$ at $4 \mathrm{~K}^{30}$. The white outlines show the $\mathrm{MoSe}_{2} / \mathrm{WSe}_{2}$ heterostructure area. The laser excitation spot is fixed at the top left of the sample. Scale bar, $5 \mu \mathrm{m}$. c Normalized PL intensity of interlayer excitons versus distance from the excitation point under different excitation powers ${ }^{30}$. $\mathbf{d}$ Time-dependent electroluminescence (EL) intensity of the neutral (orange, $150 \mathrm{~ns}$ ) and charged (blue, $\sim 25 \mathrm{~ns}$ ) interlayer excitons in the $\mathrm{MoSe}_{2} / \mathrm{WSe}_{2}$ heterobilayer ${ }^{30}$. e EL energy of the interlayer exciton versus the electric field $\left(E_{\mathrm{hs}}\right)$ applied vertically to the $\mathrm{MoSe}_{2} / \mathrm{WSe}_{2}$ heterostructure ${ }^{30}$. a-e Reprinted with permission from ref. ${ }^{30}$ [American Association for the Advancement of Science]

repulsion. At the applied highest laser power, the interlayer excitons could diffuse several micrometers away from the excitation $\operatorname{spot}^{34}$ or even across the whole sam$\mathrm{ple}^{30}$. Additionally, when free charge carriers were introduced by electrostatically doping one of the TMD layers, charged interlayer excitons that could be controlled by an in-plane electric field were observed, and they could also drift across the entire heterostructure $\left(\mathrm{MoSe}_{2} / \mathrm{WSe}_{2}\right)$ under an applied bias voltage ${ }^{30}$. More intriguingly, in this $\mathrm{MoSe}_{2} / \mathrm{WSe}_{2}$ heterobilayer, the electrical generation of both long-lived neutral ( 150 ns) and charged ( $25 \mathrm{~ns})$ interlayer excitons was realized by free-carrier injection using ohmic contacts in individual monolayers, and the electroluminescence (EL) energy of the interlayer excitons could be tuned in ranges of over hundreds of $\mathrm{meV}$ with the external electric field (Fig. 6d, e) ${ }^{30}$, similar to the behavior of PL emission due to the Stark effect. This achievement is meaningful since an electrically driven near-infrared light source, in addition to signal processing devices relying on transport characteristics, is highly desirable in optoelectronic integrated circuits.
The above investigations focused on interlayer exciton transport at low temperature, while practical applications prefer devices operating at room temperature. The upper bound of the temperature $(T)$ where excitons can exist can be roughly determined by $E_{\mathrm{b}} / k_{\mathrm{B}}$, where $E_{\mathrm{b}}$ is the exciton binding energy and $k_{\mathrm{B}}$ is the Boltzmann constant ${ }^{172,186,187}$. In traditional semiconductor CQWs, the resulting indirect excitons usually have a small $E_{\mathrm{b}}$ of several to tens of meV $\left(<k_{\mathrm{B}} T\right.$ in most cases), resulting in excitonic devices generally operating at cryogenic temperatures ${ }^{172,187,188}$. However, the interlayer excitons formed in TMD vdW heterostructures have a much larger $E_{\mathrm{b}}$, with reported values exceeding $100 \mathrm{meV}^{17,21,42,55,87,81-86,88,89}$, leading to a much higher predicted temperature $(>1000 \mathrm{~K})$, which facilitates the realization of excitonic devices that operate at room temperature. In fact, excitonic devices based on an hBN-encapsulated $\mathrm{MoS}_{2} / \mathrm{WSe}_{2}$ heterobilayer with electrically controlled transistor actions at room temperature were realized recently ${ }^{29}$. In the vertical direction of this heterostructure, multiple transparent graphene electrodes (gates 1-3, gate voltages $V_{\mathrm{g} 1}-V_{\mathrm{g} 3}$ ) were fabricated to 


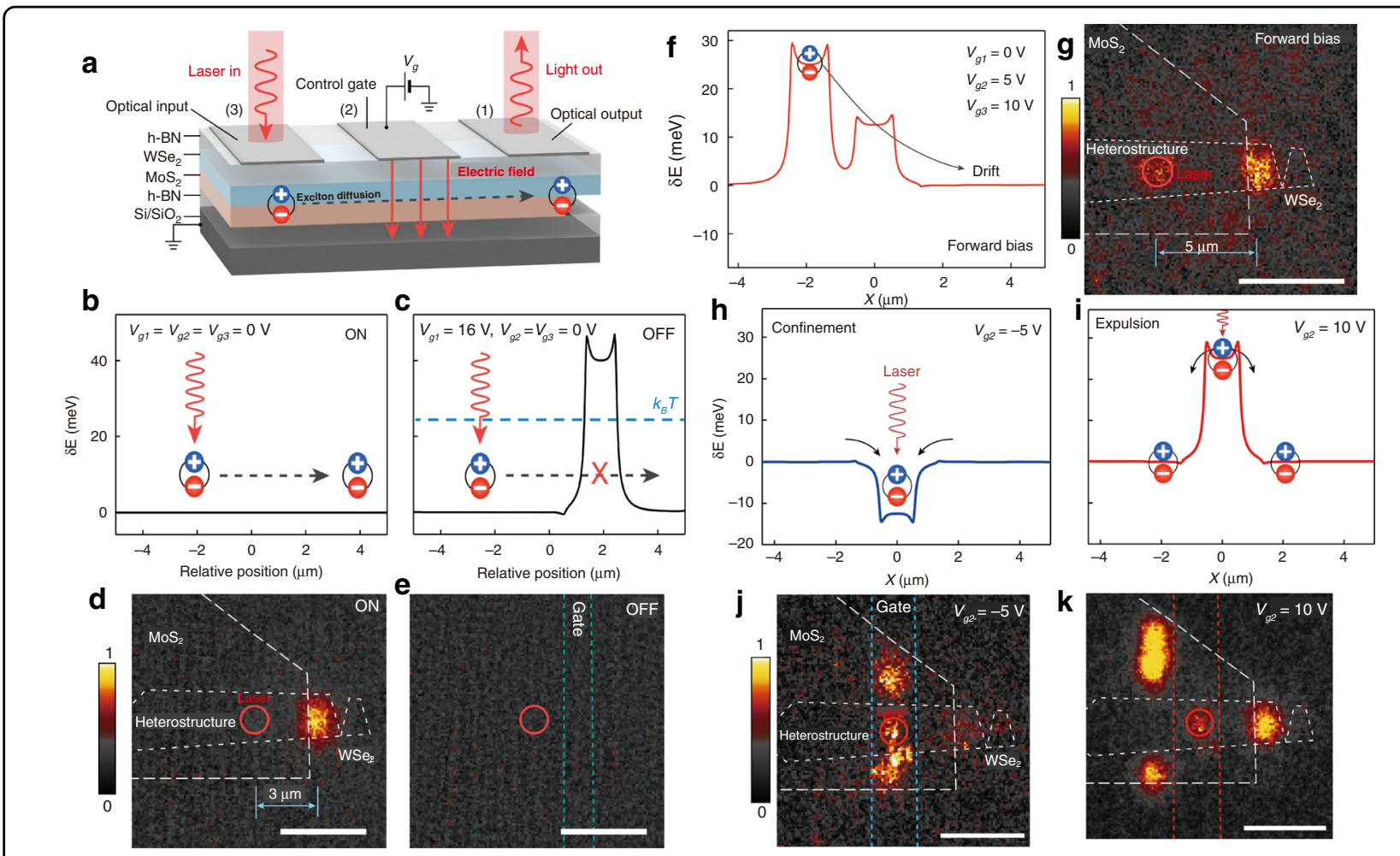

Fig. 7 Control of the interlayer exciton transport by a laterally modulated potential landscape at room temperature. a Schematic illustration of the $\mathrm{MoS}_{2} / \mathrm{WSe}_{2}$ heterostructure encapsulated in hexagonal boron nitride ( $\left.\mathrm{h}-\mathrm{BN}\right)$ with top and bottom gates ${ }^{29}$. The three gate voltages $\left(V_{\mathrm{g} 1}, V_{\mathrm{g} 2}\right.$ and $V_{g_{3}}$ ) applied to the transparent graphene electrodes (gates 1-3) can be engineered to provide a potential landscape for controlling the interlayer exciton transport through the device. $\mathbf{b}, \mathbf{c}$ Calculated energy variation $\delta$ E for the interlayer excitons in the $\mathrm{ON}$ ( $\mathbf{b}$ free diffusion, $V_{\mathrm{g} 1}=V_{\mathrm{g} 2}=V_{\mathrm{g} 3}=0 \mathrm{~V}$ ) and OFF (c potential barrier, $V_{\mathrm{g} 1}=16 \mathrm{~V}, V_{\mathrm{g} 2}=V_{\mathrm{g} 3}=0 \mathrm{~V}$ ) states $^{29}$. Red arrows represent laser excitation, and black dashed arrows denote interlayer exciton diffusion. $\mathbf{d}$, e Corresponding images of the interlayer exciton emission in the ON and OFF states ${ }^{29}$. Dashed lines denote the positions of the $\mathrm{MoS}_{2}$ and $\mathrm{WSe}_{2}$ monolayers and the top graphene gate (gate 1). The red circle represents the laser spot. Scale bars, $5 \mu \mathrm{m}$. $\mathbf{f}$ Calculated energy profile $\delta \mathrm{E}$ of the interlayer exciton as a function of the lateral coordinate $X$ under the forward bias case $\left(V_{\mathrm{g} 1}=0 \mathrm{~V}, V_{\mathrm{g} 2}=5 \mathrm{~V}, V_{\mathrm{g} 3}=10 \mathrm{~V}\right)^{29}$. The black solid lines show the direction of interlayer exciton drift. $\mathbf{g}$ Image of the interlayer exciton emission under the forward bias in $\mathbf{f}$, demonstrating a drift distance of $\sim 5 \mu \mathrm{m}^{29}$. Scale bars, $5 \mu \mathrm{m}$. $\mathbf{h}, \mathbf{i}$ Calculated energy profile $\delta E$ of the interlayer exciton for the cases of a potential well (h confinement) and a potential barrier (i, expulsion) $)^{29}$. j, $\mathbf{k}$ Images of the interlayer exciton emission for the potential landscapes shown in $\mathbf{h}$ and $\mathbf{i}^{29}$. Scale bars, $5 \mu$ m. a-k Reprinted with permission from ref. ${ }^{29}$ [Springer Nature Limited]

provide a laterally modulated potential landscape to control the exciton flux (Fig. $7 \mathrm{a})^{29}$. In the absence of electric fields $\left(V_{\mathrm{g} 1-\mathrm{g} 3}=0\right)$, the interlayer excitons could freely diffuse away from the laser spot by approximately $3 \mu \mathrm{m}$ and reach the recombination site with a bright emission output (marked as the "ON" state for the excitonic switch, Fig. 7b, d). Note that during this diffusion process, the interlayer excitons were expected to dissociate into single carriers that could diffuse inside monolayer $\mathrm{WSe}_{2}$ and $\mathrm{MoS}_{2}$ to recombine with the native charges ${ }^{29}$. However, when introducing a potential barrier higher than $k_{\mathrm{B}} T$ on the diffusion path (i.e., $V_{\mathrm{g} 1}=+16 \mathrm{~V}, V_{\mathrm{g} 2, \mathrm{~g} 3}=0$ ), the interlayer exciton transport was impeded by the barrier, causing a dark output (marked as the "OFF" state for the excitonic switch, Fig. 7c, e). Therefore, a change in the $\mathrm{ON}$ and OFF states in the excitonic switch could be realized by selectively introducing a barrier to either allow or block exciton transport. The intensity ratio of the ON and OFF states was reported to be larger than 100 at room temperature. In addition, an upwards or downwards potential gradient could be laterally created by using all the electrodes to further manipulate the exciton transport. For instance, the interlayer exciton transport could be enhanced (over $5 \mu \mathrm{m}$ ) under a progressively lower energy profile due to the addition of drift motion along the path (Fig. 7f, g). Moreover, various types of potential landscapes, such as a potential well (Fig. $7 \mathrm{~h}, \mathrm{j}$ ) or a repulsive barrier (Fig. 7i, k), could be created by applying appropriate voltages to either trap the interlayer excitons in the well or allow them to drift away from the barrier, opening a route to versatilely manipulate the exciton transport in future excitonic devices. Room-temperature exciton transport was also found in another $\mathrm{MoS}_{2} / \mathrm{WSe}_{2}$ heterobilayer stacked on a silicon-on-insulator substrate with a 
silicon suspended slab in the middle ${ }^{136}$. Intriguingly, this unique suspended slab geometry introduced a strong potential gradient for the interlayer excitons, combined with their long recombination lifetimes, both of which enabled the observation of the valley Hall effect even at room temperature ${ }^{136}$.

\section{Valley-polarized interlayer exciton transport}

Compared to the indirect excitons formed in traditional semiconductor CQWs, another advantage of the interlayer excitons in TMD vdW heterostructures is their inherited valley-contrasting physics. As discussed before, interlayer excitons have long valley-polarized lifetimes as well, ranging from nanoseconds to microseconds ${ }^{27,33}$, which has motivated scientists to explore the transport properties of valley-polarized interlayer excitons. An early work demonstrated that the lateral drift and diffusion of valleypolarized interlayer excitons could reach several micrometers owing to their long valley-polarized lifetimes $(\sim 40 \mathrm{~ns})^{33}$. The spatial pattern of the valley polarization for the interlayer excitons evolved into a ring with a diameter that increased with the excitation intensity (Fig. 8a) ${ }^{33}$, which was strikingly different from the spatial pattern of unpolarized interlayer excitons stated above. Since exciton diffusion due to a density gradient is valley-independent and unlikely to generate a ring pattern, the observed ring pattern in the valley polarization was ascribed to valleydependent exciton exchange interactions that caused the majority valley excitons to experience a stronger repulsive force, and thus more rapid expansion than the minority excitons leading to valley-asymmetric transport of the interlayer excitons with the resulting ring pattern in the spatial distribution $^{33,35}$.

Similar to the aforementioned excitonic switch based on the manipulation of interlayer exciton transport, an excitonic switch based on valley-polarized interlayer excitons was also recently realized at low temperatures (4 and $100 \mathrm{~K})^{34}$. The ON and OFF states of this switch were achieved by removing and introducing a potential barrier on the transport path, and an approximately $1.4 \mu \mathrm{m}$ difference in the diffusion distance of the valley-polarized interlayer excitons was observed between the ON and OFF states (Fig. 8b-g) ${ }^{34}$. Analogously, the transport behavior (repulsive drift or confinement trap) of the valley-polarized interlayer excitons could be manipulated by designing various potential profiles, such as a potential barrier or well along the travel path (Fig. $8 \mathrm{~h}-\mathrm{j})$. Very interestingly, when a potential well was applied to trap valley-polarized interlayer excitons, the exciton concentration could be increased by an order of magnitude (a lower bound of $\sim 1.8 \times 10^{12} \mathrm{~cm}^{-2}$ ), which was a promising step towards the realization of a macroscopic quantum state of valley-polarized excitons via Bose-Einstein condensation $^{34}$. Further work is expected to achieve the accumulation of valley-polarized excitons at higher temperatures by engineering or optimizing the potential profiles in these systems.

Notably, single particles such as resident holes in holedoped heterostructures can serve as efficient valley information carriers as well, with a reported spin-valley diffusion current persisting for more than $20 \mu \mathrm{s}$ accompanied by a travel distance of over $20 \mu \mathrm{m}^{170}$. A detailed description of this topic is beyond the scope of this review.

\section{Interlayer exciton transport under moiré potentials}

In TMD vdW heterostructures, the potential landscape for manipulating the interlayer exciton transport can be modified not only by external electric fields but also by an internal moiré pattern, which introduces a twist-angledependent moiré potential on the interlayer excitons. Thus far, the above discussed interlayer exciton transport has focused on the exciton motion under externally applied electric fields. Experimental investigations on how excitons move in a potential landscape modulated by moiré patterns are rare.

Recently, the role of moiré potentials in exciton transport was experimentally addressed using transient absorption microscopy (TAM) combined with first-principles calculations ${ }^{91}$. In this study ${ }^{91}, \mathrm{WS}_{2} / \mathrm{WSe}_{2}$ heterobilayers with two different twist angles $\left(0\right.$ and $\left.60^{\circ}\right)$ were fabricated to study the effect of the twist-angle-dependent moiré potential on exciton transport (Fig. 9a-c). The moiré potentials of $0^{\circ}$ and $60^{\circ}$ heterobilayers were first theoretically calculated (Fig. 9d-g), with the spatial variations for $0^{\circ}$ being much stronger (deep potential) than those for $60^{\circ}$ (shallow potential), implying that the interlayer excitons encountered a higher energy barrier as they moved from one location to another in the $0^{\circ}$ heterobilayers. In other words, the interlayer excitons in $60^{\circ}$ heterobilayers should be more mobile due to less confinement from the shallow potential. This was further proven by imaging the spatiotemporaldependent exciton population via the TAM technique, from which the time-dependent mean squared distances $\left(\sigma_{\mathrm{t}}^{2}-\sigma_{0}^{2}\right)$ traveled by both inter- and intralayer excitons were obtained (Fig. 9h). Deviating from the normal diffusion behavior of intralayer excitons with a linear temporal dependence on the mean squared distance, anomalous diffusion of interlayer excitons, with a nonlinear temporal dependence on the mean squared distance and a faster diffusion speed, was observed for both $0^{\circ}$ and $60^{\circ}$ heterobilayers, suggesting that the repulsive interlayer exciton interactions played a central role in their diffusion process. As expected, the interlayer excitons in $60^{\circ}$ heterobilayers traveled faster than those in $0^{\circ}$ heterobilayers, consistent with the prediction from calculations and strongly indicating the role of moiré potentials in mediating exciton transport. The interlayer excitons were also transported faster at higher excitation densities owing to the repulsive interactions between the interlayer excitons (Fig. 9i). 


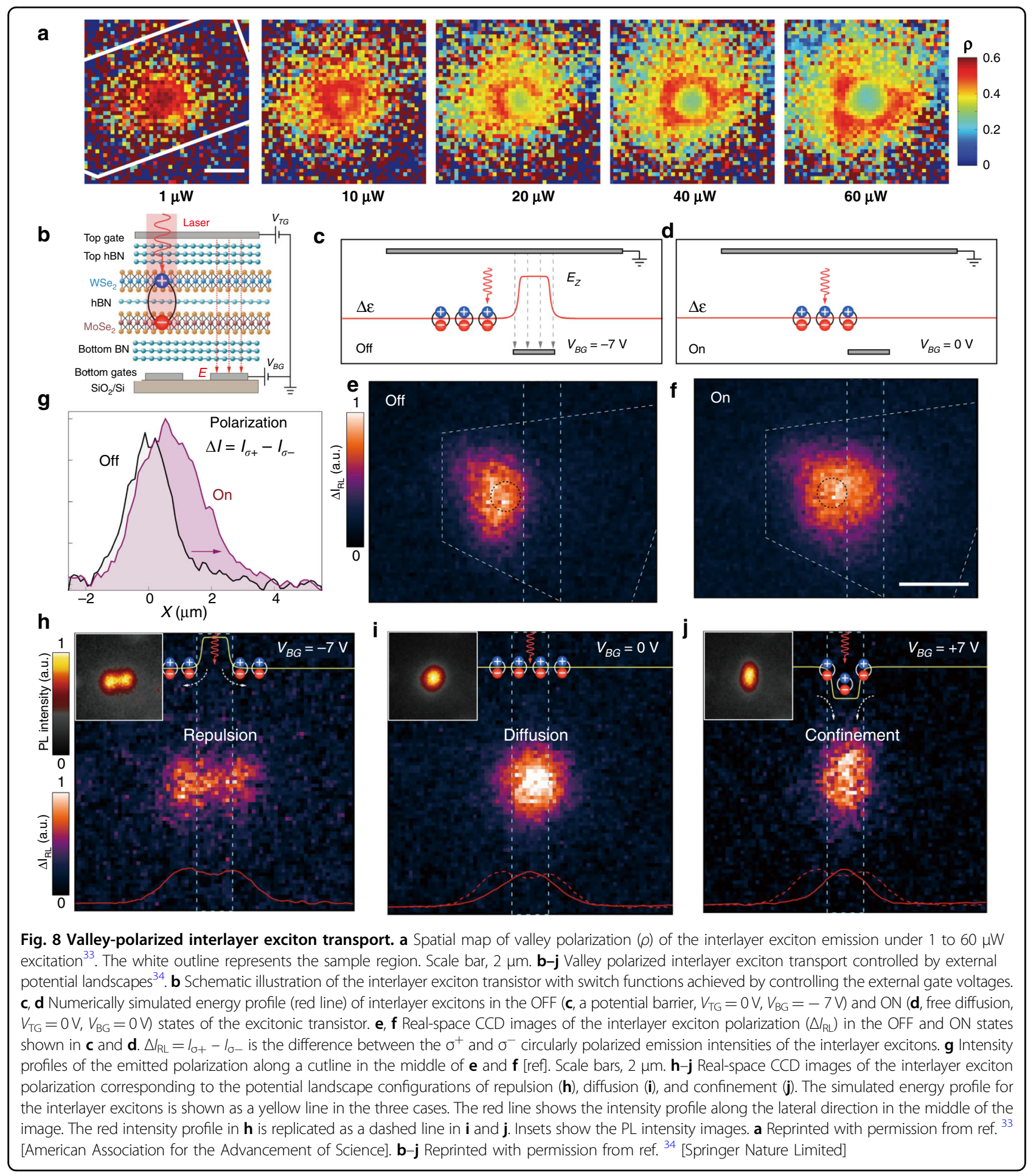

The effect of moiré potentials on exciton transport was further supported by temperature-dependent measurements. As observed, the exciton transport speed increased as the temperature increased (Fig. $9 \mathrm{j})^{91}$ owing to the more effective screening of the moiré potentials at higher temperatures. This observation is seemingly inconsistent with the temperature-dependent steady-state PL measurements, with the interlayer excitons transported over longer distances at lower temperatures ${ }^{29,30,34}$. However, steady-state measurements are integrated over the entire exciton lifetime, and the slower exciton transport at lower temperatures could be compensated by the corresponding 


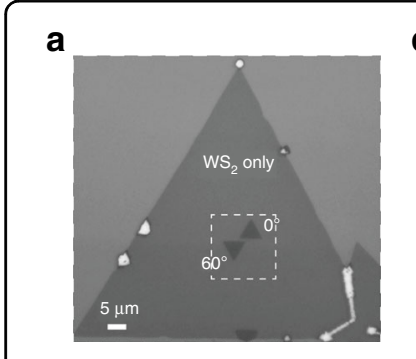

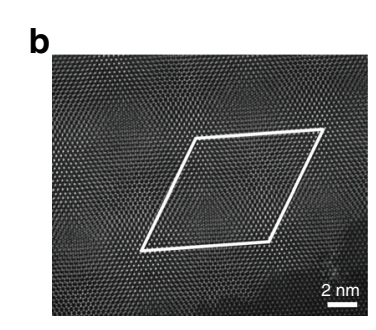
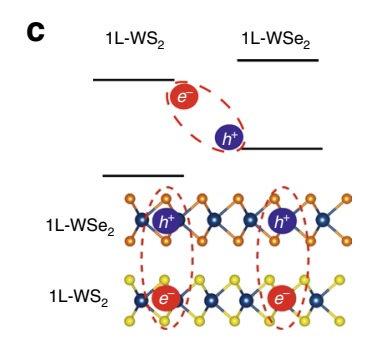

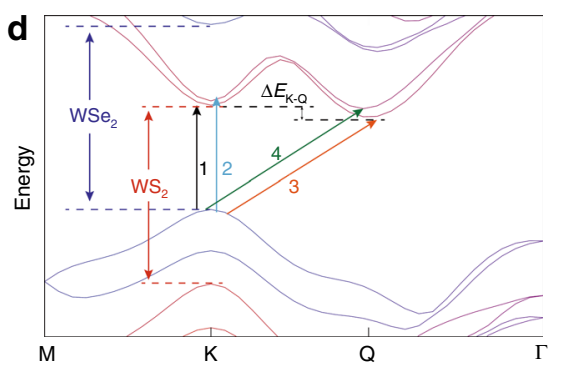

$\mathbf{f}$
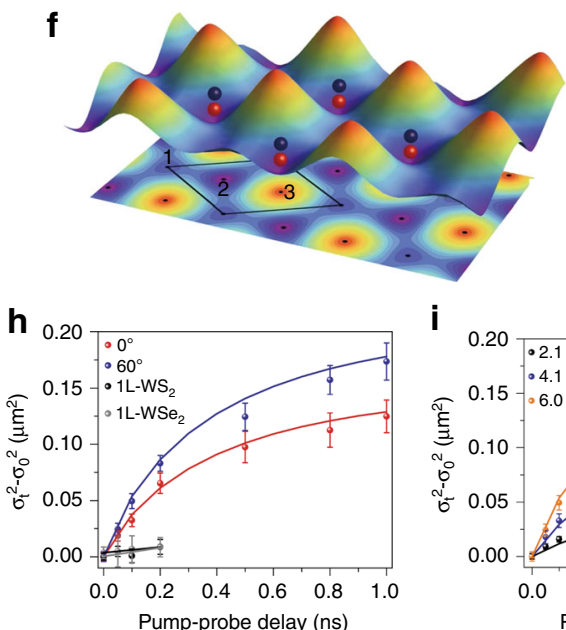

i
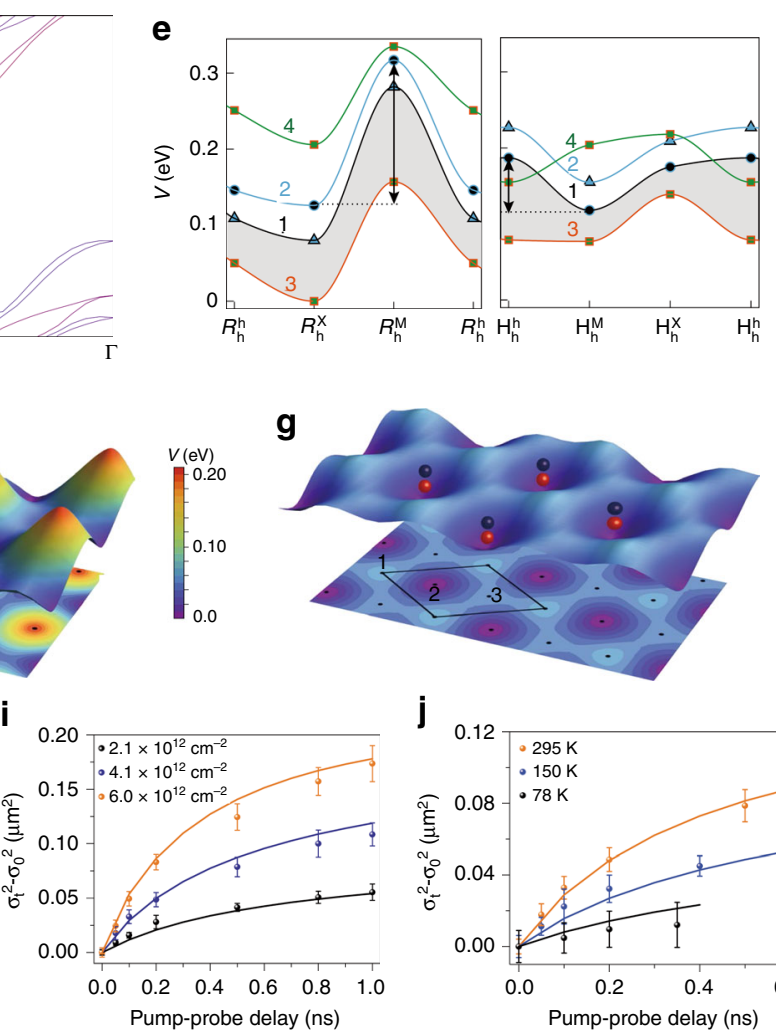

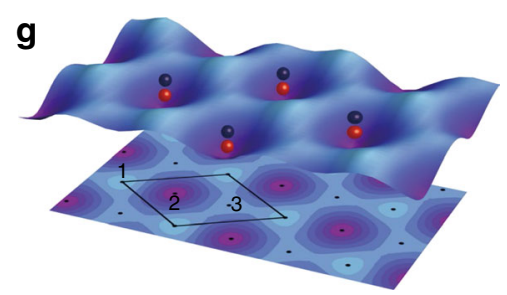

j

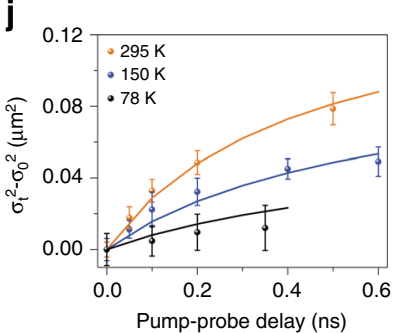

Fig. 9 Interlayer exciton transport under a moiré potential. a Optical image of two CVD-grown WS $\mathrm{WWS}_{2}$ heterobilayers with twist angles of 0 and $60^{\circ}$ on the same $W_{2}$ underlayer ${ }^{91}$. b High-resolution annular dark-field scanning transmission electron microscopy image of a $60^{\circ}$ heterobilayer ${ }^{91}$. The white diamond outline shows a moiré superlattice with a periodicity of $\sim 7.6 \mathrm{~nm}$. c Schematic illustration of the WS $\mathrm{SWSe}_{2}$ heterobilayer with a type-II band alignment for facilitating interlayer exciton formation ${ }^{91}$. $\mathbf{d}$ Schematic representation of the typical electronic band structure of a $\mathrm{WS}_{2} \mathrm{WSe}_{2}$ heterobilayer in a (strained) primitive unit cell ${ }^{91}$. The four lowest-energy transitions are indicated by arrows (K-K valley transitions are denoted by vertical arrows 1 and 2, and $\mathrm{K}-\mathrm{Q}$ valley transitions are denoted by vertical arrows 3 and 4). The $\mathrm{K}-\mathrm{K}$ transitions in individual $\mathrm{WS}_{2}$ and $\mathrm{WSe}_{2}$ monolayers are marked by vertical arrows $\mathrm{WS}_{2}$ and $\mathrm{WSe}_{2}$, respectively. e Approximate moiré potentials for twist angles of $0^{\circ}$ (left) and $60^{\circ}$ (right) plotted along the main diagonal of the moiré supercells (black lines in $\left.\mathbf{f}\right)^{91}$. The spatial potential variations for $0^{\circ}$ are much stronger (deep potential) than those for $60^{\circ}$ (shallow potential) heterobilayers. The different lines correspond to the four lowest-energy optical transitions marked in d. f, $\mathbf{g}$ Illustrations of both $3 \mathrm{D}$ graphs and 2D projections of the 2D K-K moiré potentials for trapping interlayer excitons (red and black spheres) in local minima for $0^{\circ}(\mathbf{f})$ and $60^{\circ}(\mathbf{g})$ heterobilayers ${ }^{91}$. A twist-angle-dependent moiré potential is indicated by the theoretical results. The numbers 1,2 , and 3 indicate the three high-symmetry local atomic registries in a moiré superlattice. $\mathbf{h}$ Time-dependent mean squared distances $\left(\sigma_{t}{ }^{2}-\sigma_{0}{ }^{2}\right)$ traveled by interlayer excitons in $0^{\circ}$ and $60^{\circ}$ heterobilayers as well as by intralayer excitons in $\mathrm{WS}_{2}$ and $\mathrm{WSe}_{2} \mathrm{monolayers}\left(1 \mathrm{~L}-\mathrm{WS}_{2} \text {, } 1 \mathrm{~L}-\mathrm{WSe}_{2}\right)^{91}$. $\mathbf{i}$ Densitydependent interlayer exciton transport at room temperature for the $60^{\circ}$ heterobilayer ${ }^{91}$. $\mathbf{j}$ Temperature-dependent interlayer exciton transport for the $60^{\circ}$ heterobilayer ${ }^{91}$. a-j Reprinted with permission from ref. ${ }^{91}$ [Springer Nature Limited]

longer lifetimes, which could lead to an overall longer transport distance at lower temperatures ${ }^{91}$.

Similar to the former discussed work, interlayer exciton transport under periodic moiré potential in both R-type and $\mathrm{H}$-type $\mathrm{MoSe}_{2} / \mathrm{WSe}_{2}$ heterostructures was studied later, from which the diffusion barrier experienced by the interlayer excitons was experimentally quantified by studying the exciton transport at various temperatures ${ }^{189}$. The influence of the moiré potential on interlayer exciton diffusion was also studied recently via spatially and spectrally resolved PL imaging in $\mathrm{MoSe}_{2} / \mathrm{WSe}_{2}$ heterostructures with different twist angles (Fig. 10a-f) ${ }^{162}$. In this study, three types of heterostructures were used, with the first prepared by the CVD method with a twist angle of $0^{\circ}$ (no moiré pattern) and the second and third prepared by the mechanical exfoliation and transfer (MET) method with twist angles of $\sim 1.1^{\circ}$ (moiré period of $\sim 17 \mathrm{~nm}$ ) and $\sim 3.5^{\circ}$ (moiré period of $\sim 5 \mathrm{~nm}$ ), respectively. The interlayer exciton diffusion of these samples was remarkably different (Fig. 10a-f). The diffusion length in the CVD-grown sample with no moiré potential was the longest (a few microns), exceeding the boundaries of the heterostructure region. The diffusion in the heterostructure with a twist angle of $\sim 3.5^{\circ}$ (a moiré period of $\sim 5 \mathrm{~nm}$ ) was finite, with a diffusion length of $\sim 1 \mu \mathrm{m}$, while no diffusion beyond the laser spot was observed in the heterostructure with a relatively smaller twist angle $\left(\sim 1.1^{\circ}\right)$ but a larger moiré period $(\sim 17 \mathrm{~nm})$. These results suggested that a moiré 


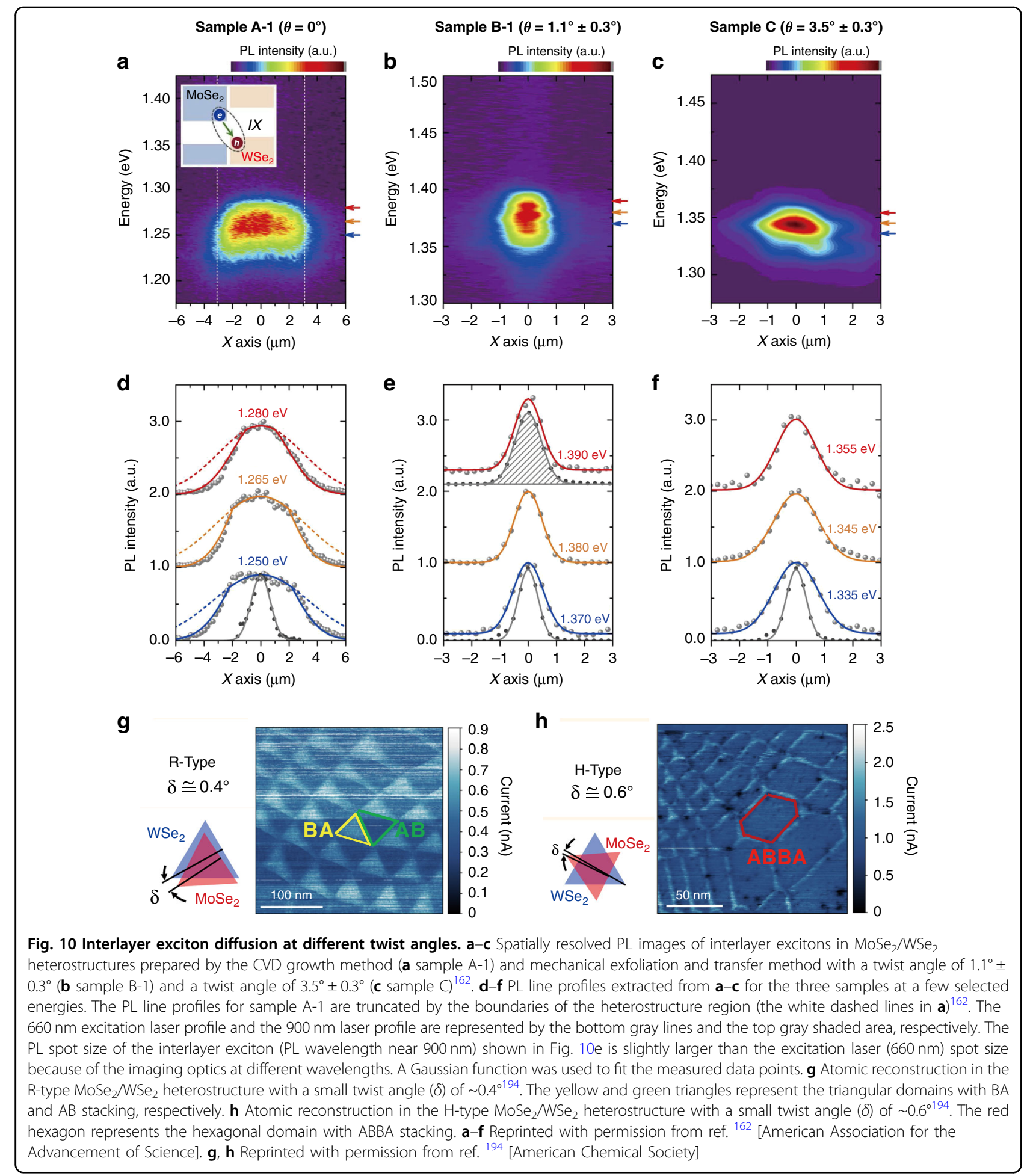

superlattice has a profound effect on interlayer exciton diffusion. As stated ${ }^{162}$, diffusion in CVD-grown commensurate heterostructures with no moiré pattern should be the most free and reach the longest distance. In contrast, in heterostructures with a larger moiré period (a smaller twist angle), the interlayer excitons could be substantially localized by the moiré potential without obvious diffusion. For heterostructures with a smaller moiré period (a larger twist angle and a longer interlayer exciton lifetime due to the larger momentum mismatch), interlayer excitons may tunnel between the supercells and diffuse a finite distance over a longer interlayer exciton 
lifetime. All these works indicate that the diffusion barrier introduced by the moiré potential could be modified to affect exciton migration by tuning the twist angle or stacking mode between the constituent monolayers, which offers a novel way to control the exciton transport behavior in potential excitonic devices.

However, note that when the twist angle between layers was rather small (generally less than $1^{\circ}$ ), atomic reconstruction could take place and was expected to have a profound effect on the band structures of the twisted bilayers $^{190-193}$. In this case, the continuously varying rigidlattice moiré pattern transformed to discrete commensurate domains divided by narrow domain walls, which had also been recently observed in twisted TMD vdW bilayers $^{194,195}$. As reported ${ }^{194}$, in R-type heterostructures with a small twist angle of $<1^{\circ}$, atomic reconstruction could induce commensurate triangular domains with alternating $\mathrm{AB}$ and $\mathrm{BA}$ stacking configurations dominating in the whole heterostructure region $(\mathrm{AB}$ : transition metal $\mathrm{A}$ in the upper layer overlaps chalcogen $B$ in the bottom layer; $B A$ : chalcogen $\mathrm{B}$ in the upper layer overlaps transition metal A in the bottom layer), while in $\mathrm{H}$-type heterostructures with a twist angle of $<1^{\circ}$, atomic reconstruction resulted in commensurate hexagonal domains dominated by the ABBA stacking configuration (ABBA: transition metal A in the upper layer overlaps chalcogen $B$ in the bottom layer and chalcogen $B$ in the upper layer overlaps transition $A$ in the bottom layer). The average area of the hexagonal domains in the $\mathrm{H}$-type system was reported to approach twice that of the triangular domains in the R-type system $^{194}$. In other words, for atomically reconstructed bilayers with small twist angles, the high-symmetry stacking configurations that only occurred at certain sites in the rigid-lattice moiré pattern now covered almost the whole bilayer region, either in alternating $\mathrm{AB}$ and $\mathrm{BA}$ stacking (Rtype) or in ABBA stacking (H-type), which was reported to have lower stacking energies ${ }^{194}$, with their boundaries separated by very narrow domain walls (several $\mathrm{nm}^{194,195}$ ). Within these enlarged commensurate domains (several tens of $\mathrm{nm}$ or even larger than $100 \mathrm{~nm}^{194,195}$ ), the moiré potential was absent, although a diffusion barrier may have existed between the boundaries. Therefore, the potential landscape imposed on the interlayer excitons of a moiré heterostructure with a relatively large twist angle may vary notably from those of an atomic reconstructed heterostructure with a very small twist angle. Correspondingly, the scenarios of the interlayer exciton transport may be different for these two classes of heterostructures. These differences may even exist between the R-type and H-type heterostructures after atomic reconstruction due to their remarkably different rearranged configurations. Further theoretical and experimental investigations are expected to reveal these discrepancies and to provide deep insight into this new type of heterostructure.

\section{Applications}

After a detailed description of the interlayer exciton formation, relaxation, and transport properties in TMD $\mathrm{vdW}$ heterostructures, a brief introduction of its potential applications is presented in this section. According to the above discussion, interlayer excitons have a number of excellent characteristics such as (1) high exciton binding energy (>100 meV), (2) energy tuneable by an external electric field, (3) long population and valley-polarized lifetimes, (4) long-range transport, and (5) the potential to realize high-temperature Bose-Einstein condensates. All these factors make interlayer excitons very suitable for developing excitonic devices as the counterpart of electronic devices for signal processing ${ }^{173}$, and such excitonic signal processing devices have the following advantages: first, interlayer excitons and photons can directly transform into each other, allowing efficient coupling of the signal processing to optical communication, especially in potential silicon-based optoelectronics; second, interlayer excitons can act as spin-valley information carriers due to the inherited spin-valley properties, enabling efficient signal processing with reduced power consumption; and third, interlayer excitons hold great promise for realizing a high-temperature coherent condensate ${ }^{32}$, making the creation of an exciton current with no resistance possible in future computation.

Various excitonic signal processing devices such as excitonic transistors, routers, and photon storage devices have been explored based on indirect excitons in traditional semiconductor CQWs ${ }^{173}$, which motivates investigations of excitonic devices based on interlayer excitons in TMD vdW heterostructures. As stated above, excitonic transistors with switch functions from the manipulation of the interlayer exciton transport have been achieved by proof-of-principle in TMD vdW heterostructures ${ }^{29,34}$. An excitonic switch through exchange of the emission polarization of interlayer excitons has also been recently demonstrated in a $\mathrm{MoSe}_{2} / \mathrm{WSe}_{2}$ heterostructure $^{117}$. The difference between right and left circularly polarized emission intensities $\left(\Delta I_{\mathrm{RL}}\right)$ as a function of the gate voltage $\left(V_{\mathrm{TG}}\right)$ was studied in this work to demonstrate the polarization switching actions (Fig. 11a) ${ }^{117}$. For $V_{\mathrm{TG}}$ below $0 \mathrm{~V}$ or higher than $5 \mathrm{~V}$, an "inverter" reversing the input polarization (negative $\Delta I_{\mathrm{RL}}$ ) was obtained. However, for $V_{\mathrm{TG}}$ between 0 and $5 \mathrm{~V}$, a "transmitter" preserving the input polarization (positive $\Delta I_{\mathrm{RL}}$ ) could be achieved. Such electrical manipulation of the emission polarization of interlayer excitons paves the way for their practical applications in signal processing and integrated optoelectronics.

In addition to signal processing devices, other devices, especially light sources and photodetectors, are keystone components in integrated optoelectronics or siliconbased optoelectronics. Very encouragingly, excitonic 


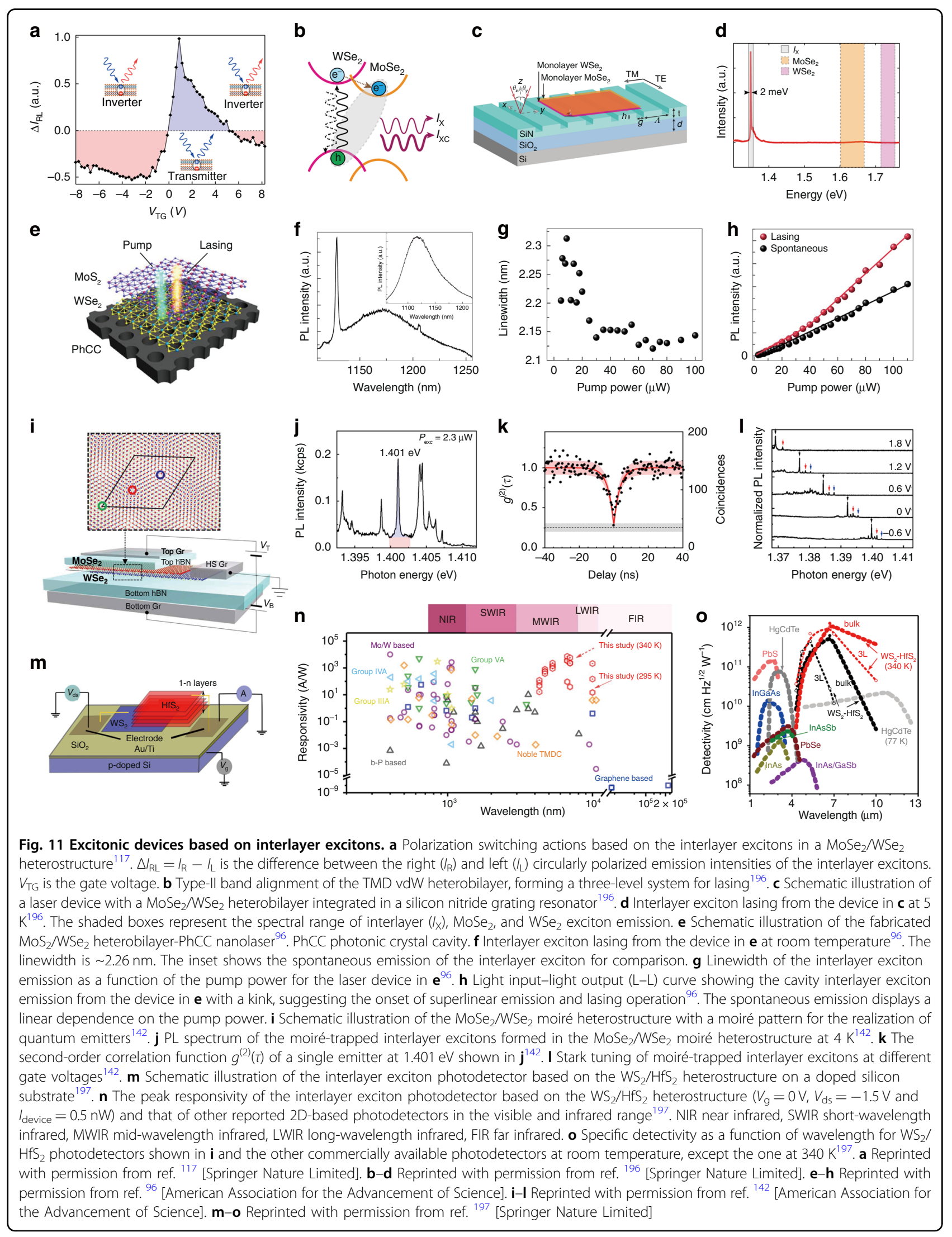


lasers and photodetectors based on interlayer excitons were recently realized in TMD vdW heterostructures $^{96,196,197}$. Owing to the ultrafast charge transfer and the long lifetimes of the formed interlayer excitons, the type-II band aligned heterostructure actually provides a three-level system to facilitate the establishment of a population inversion for lasing (Fig. 11b) ${ }^{196}$. Through this, spatially coherent lasing emission from direct-bandgap interlayer excitons was realized in a rotationally aligned $\mathrm{MoSe}_{2} / \mathrm{WSe}_{2}$ heterobilayer integrated in a silicon nitride grating resonator at low temperature (Fig. 11c, d) ${ }^{196}$. Later, a roomtemperature near-infrared laser based on interlayer excitons in a $\mathrm{MoS}_{2} / \mathrm{WSe}_{2}$ heterobilayer on a silicon photonic crystal cavity was achieved (Fig. 11e-h) ${ }^{96}$. These works indicate that interlayer excitons in TMD vdW heterostructures can act as an efficient gain medium to support lasing emission, which may offer new opportunities for developing coherent light sources with desired optical properties for integrated optoelectronics. Moreover, quantum light based on moiré-trapped interlayer excitons in a $\mathrm{MoSe}_{2} / \mathrm{WSe}_{2}$ heterostructure has been reported recently (Fig. $11 \mathrm{i}-\mathrm{l})^{142}$, although the emission from a single moiré unit cell was not specified. In this study, the moiré-trapped interlayer excitons manifested as several discrete sharp lines in the PL spectrum, with extraordinarily narrow linewidths of several tens to hundreds of $\mu \mathrm{eV}$ (Fig. 11j). The quantum nature of the moiré-trapped interlayer excitons was established via the observation of photon antibunching (Fig. 11k). In addition, the emission energy of these quantum emitters could be tuned up to $40 \mathrm{meV}$ through the direct current Stark effect (Fig. 11l). This study provides a route for investigating and understanding the nature of moiré quantum emitter arrays. On the other hand, photodetectors, serving as optical to electrical converters, are also an essential component of integrated optoelectronics. A highly responsive room-temperatureoperated infrared photodetector, with detection range tuning from mid-wavelength to long-wavelength infrared (MW-LWIR) under a modest electric field, was realized recently based on interlayer excitons in a $\mathrm{WS}_{2} / \mathrm{HfS}_{2}$ heterostructure (Fig. 11m) ${ }^{197}$. The responsivity of this MWLWIR photodetector was reported to be comparable to those of Mo/W-based photodiodes in the visible and near infrared range (Fig. 11n), and the calculated detectivities $\left(D^{*}\right)$ of this photodetector were shown to be higher than those of other commercially available infrared photodetectors, especially for room and elevated temperature operations (Fig. 110). These findings demonstrate the great promise of interlayer excitons for versatile optoelectronic applications.

Nevertheless, research on excitonic devices based on interlayer excitons in TMD vdW heterostructures is still in the early stages. Improving the performance of the already developed excitonic devices for practical applications and exploring more functional excitonic devices, such as waveguides and modulators are expected in further works. Moreover, the integration of individual excitonic devices such as light sources, switches, modulators, and detectors on a single chip is very likely and highly desirable in the future for realizing on-chip integrated optoelectronics based on two-dimensional vdW heterostructures ${ }^{96,198}$.

\section{Conclusions and outlook}

The rise of TMD vdW heterostructures has stimulated enormous interest in interlayer excitons, including their formation, relaxation, transport, and related applications. All these research topics are closely linked to each other, as discussed above. Specifically, the ultrafast charge transfer in type-II-band-aligned TMD vdW heterostructures facilitates the formation of interlayer excitons, which have a variety of fundamental characteristics such as the existence of two different kinds of electric dipoles (a reduced transition dipole and a permanent electric dipole), inherited valley-contrasting physics, and moiré configuration dependence. Benefiting from these fundamental properties, the interlayer excitons have a long population lifetime and possess significantly suppressed intervalley scattering, both of which give rise to a long valley-polarized lifetime for the interlayer excitons. As a consequence, the interlayer excitons and valley-polarized interlayer excitons have good transport characteristics, basically owing to their long lifetimes and sensitivity to external electric fields. All these factors form the basis for developing excitonic optoelectronic devices with interlayer excitons, such as excitonic transistors ${ }^{29,34}$, switches ${ }^{117}$, lasers ${ }^{96,196}$, and photodetectors $^{197}$.

Undoubtedly, as a newly controllable excitonic system, the interlayer excitons in TMD vdW heterostructures offer a very promising platform for both fundamental exploration and potential optoelectronic applications. For fundamental studies, beyond the rapid progress in revealing the intriguing features of interlayer excitons, overcoming the existing obstacles and discovering new sciences are expected to further push this area towards the central stage. For instance, a comprehensive understanding of the underlying mechanism responsible for ultrafast charge transfer with conserved spin-valley properties will lay the basis for fully clarifying the subsequent interlayer exciton formation process as well as its inherited valley-contrasting physics, which plays an important role in determining the degree of its valley polarization. Additionally, significant efforts are needed to thoroughly comprehend the valley depolarization mechanism and its relationship with the constituted materials, crystallographic alignment, stacking 
modes, doping, and external fields or potentials, aiming to unravel the interplay of these factors and achieve a sufficiently high degree of valley polarization at room temperature for practical valleytronic applications. The origins of the observed negative circular polarization and doublet interlayer excitons are also an open questions, that remain to be addressed for an in-depth understanding of the nature of interlayer excitons. Moreover, interlayer excitons offer a novel and very exciting platform for exploring excitonic Bose condensation owing to their substantial binding energies, which stabilize them at relatively high temperature; their sufficiently long lifetimes for cooling them to approach the lattice temperature; and the existing oriented or permanent electric dipoles with repulsive interactions, which prevent the formation of competing exciton complexes at high densities ${ }^{35,199,200}$. All these merits are expected to facilitate the realization of an excitonic condensate and/or superfluidity at high temperature based on interlayer excitons or even valleypolarized interlayer excitons ${ }^{31,32}$. Another intriguing topic for future exploration is moiré interlayer excitons, which have recently attracted enormous interest. Owing to their interlayer configurations, the spatially indirect interlayer excitons are expected to be significantly influenced by moiré patterns that carry periodically varied interlayer atomic registries. However, how the interlayer excitons and their properties are spatially modulated by the moiré pattern or potential remains to be intensively explored. Far-field optical spectroscopy has been applied to reflect the moiré effect on interlayer excitons ${ }^{91,141,150,152,153,155}$, while near-field optics, with a spatial resolution sufficient to resolve the intersite in a moiré superlattice, will be the more intuitive tool for selectively addressing the interlayer excitons at individual sites. In addition, it is important to know in which situations the moiré effect is favorable and in which cases it should be avoided. For instance, the moiré potential plays an important role in trapping the interlayer excitons for quantum optics ${ }^{36}$, whereas it turns to be an obstacle for driving interlayer exciton transport ${ }^{91}$. When the moiré lattice needs to be avoided in some applications, how to avoid it while preserving the other advantages of interlayer excitons is quite important. One possible solution is to use commensurate heterobilayers prepared by continuous growth via the CVD technique ${ }^{114,137,162,201}$.

For potential optoelectronic applications, as stated above, various excitonic devices such as lasers ${ }^{96,196}$, transistors $^{29,34}$ and photodetectors ${ }^{197}$ have been realized by proof-of-principle based on interlayer excitons in TMD vdW heterostructures. Specifically, light sources such as lasers based on interlayer excitons have great potential in silicon photonics and optical fiber communication systems due to the following aspects: (1) the heterostructures formed with van der Waals forces could remarkably ease the problem of lattice mismatch that restricts the material growth and integration; (2) the interlayer exciton emission wavelength is tuneable by either the constituted materials or external electric field to be compatible with the silicon photonics or optical fiber communication band; and (3) the use of the type-II band alignment to form the interlayer excitons provides a natural three-level system, providing the possibility of population inversion for achieving coherent light sources. Such advantages promise a bright future for interlayer excitons in photonic applications. More efforts will be dedicated to improving the performance of the developed light sources such as increasing the absorption/emission of the interlayer excitons by coupling with plasmonics, creating hybrid interlayer excitons with inherited brightness from the intralayer counterparts, and designing multilayer stacking modes to increase both the interlayer coupling and the absorbance. Additionally, electrically pumped light sources will be highly desirable and will very likely be realized with interlayer excitons for integrated optoelectronic applications. Similar strategies could be applied for valley-polarized interlayer excitons to realize polarized light sources carrying valley information. Further, quantum emitters based on interlayer excitons will be a promising direction for future exploration. Various potential minima may be constructed by electric fields, strains, or especially moiré patterns to trap either one or two interlayer excitons ${ }^{142}$, enabling the realization of single photon sources or entangled-photon sources for potential quantum computation and information processing. Other excitonic devices such as transistors operating in switching mode have been reported with interlayer excitons ${ }^{29,34}$, while such devices operating in amplification mode are still lacking and highly desirable for practical signal processing. Moreover, more functional excitonic devices such as waveguides and modulators await exploitation to further extend and promote practical applications based on interlayer excitons. More intriguingly, it is expected that the full integration of these individual excitonic devices, such as light sources, waveguides, modulators, and detectors, on a single chip to realize on-chip integrated optoelectronics based on interlayer excitons will be highly possible in the future.

\section{Acknowledgements}

The authors are grateful to the National Natural Science Foundation of China (Nos. 52072117, 21703059, 51972105, 51525202, 61635001, and 61905071), the Joint Funds of the National Natural Science Foundation of China (No. U19A2090), the Key Program of the Hunan Provincial Science and Technology Department (No. 2019XK2001), the International Science and Technology Innovation Cooperation Base of Hunan Province (2018WK4004), and the Open Project Program of Wuhan National Laboratory for Optoelectronics (No. 2020WNLOKF002)

Conflict of interest

The authors declare no competing interests. 
Received: 21 October 2020 Revised: 8 February 2021 Accepted: 24 February 2021

Published online: 02 April 2021

\section{References}

1. Zeng, H. L. \& Cui, X. D. An optical spectroscopic study on two-dimensional group-VI transition metal dichalcogenides. Chem. Soc. Rev. 44, 2629-2642 (2015).

2. Yu, H. Y. et al. Valley excitons in two-dimensional semiconductors. Natl Sci. Rev. 2, 57-70 (2015).

3. $\mathrm{Xu}, \mathrm{X}$. D. et al. Spin and pseudospins in layered transition metal dichalcogenides. Nat. Phys. 10, 343-350 (2014).

4. Mak, K. F., Xiao, D. \& Shan, J. Light-valley interactions in $2 \mathrm{D}$ semiconductors. Nat. Photonics 12, 451-460 (2018).

5. Liu, Y. P. et al. Valleytronics in transition metal dichalcogenides materials. Nano Res. 12, 2695-2711 (2019).

6. Geim, A. K. \& Grigorieva, I. V. Van der Waals heterostructures. Nature 499, 419-425 (2013).

7. Novoselov, K. S. et al. 2D materials and van der Waals heterostructures. Science 353, aac9439 (2016).

8. Liu, Y. et al. Van der Waals heterostructures and devices. Nat. Rev. Mater. 1, 16042 (2016).

9. Gong, C. et al. Band alignment of two-dimensional transition metal dichalcogenides: application in tunnel field effect transistors. Appl. Phys. Lett. 103 053513 (2013).

10. Kang, J. et al. Band offsets and heterostructures of two-dimensional semiconductors. Appl. Phys. Lett. 102, 012111 (2013).

11. Guo, Y. Z. \& Robertson, J. Band engineering in transition metal dichalcogenides: stacked versus lateral heterostructures. Appl. Phys. Lett. 108, 233104 (2016)

12. Özçelik, V. O. et al. Band alignment of two-dimensional semiconductors for designing heterostructures with momentum space matching. Phys. Rev. B 94, 035125 (2016).

13. Zhang, C. X. et al. Systematic study of electronic structure and band alignment of monolayer transition metal dichalcogenides in Van der Waals heterostructures. 2D Mater. 4, 015026 (2017).

14. $\mathrm{Xu}, \mathrm{K}$. et al. The role of Anderson's rule in determining electronic, optical and transport properties of transition metal dichalcogenide heterostructures. Phys. Chem. Chem. Phys. 20, 30351-30364 (2018).

15. Chiu, M. H. et al. Determination of band alignment in the single-layer $\mathrm{MoS}_{2} /$ $\mathrm{WSe}_{2}$ heterojunction. Nat. Commun. 6, 7666 (2015).

16. Hill, H. M. et al. Band alignment in $\mathrm{MoS}_{2} / \mathrm{WS}_{2}$ transition metal dichalcogenide heterostructures probed by scanning tunneling microscopy and spectroscopy. Nano Lett. 16, 4831-4837 (2016).

17. Wilson, N. R. et al. Determination of band offsets, hybridization, and exciton binding in 2D semiconductor heterostructures. Sci. Adv. 3, e1601832 (2017).

18. Zhang, C. D. et al. Interlayer couplings, Moiré patterns, and 2D electronic superlattices in $\mathrm{MoS}_{2} \mathrm{NSS}_{2}$ hetero-bilayers. Sci. Adv. 3, e1601459 (2017).

19. Quan, C. J. et al. Band alignment of $\mathrm{MoTe}_{2} / \mathrm{MoS}_{2}$ nanocomposite films for enhanced nonlinear optical performance. Adv. Mater. Interfaces 6, 1801733 (2019).

20. Fang, H. et al. Strong interlayer coupling in van der Waals heterostructures built from single-layer chalcogenides. Proc. Natl Acad. Sci. USA 111, 6198-6202 (2014).

21. Chiu, M. H. et al. Spectroscopic signatures for interlayer coupling in $\mathrm{MoS}_{2-}$ WSe $\mathrm{S}_{2}$ van der Waals stacking. ACS Nano 8, 9649-9656 (2014).

22. Rivera, P. et al. Observation of long-lived interlayer excitons in monolayer $\mathrm{MoSe}_{2}-\mathrm{WSe}_{2}$ heterostructures. Nat. Commun. 6, 6242 (2015).

23. Miller, B. et al. Long-lived direct and indirect interlayer excitons in van der Waals heterostructures. Nano Lett. 17, 5229-5237 (2017).

24. Baranowski, M. et al. Probing the interlayer exciton physics in a $\mathrm{MoS}_{2} / \mathrm{MoSe}_{2} /$ $\mathrm{MoS}_{2}$ van der Waals heterostructure. Nano Lett. 17, 6360-6365 (2017).

25. Nagler, P. et al. Giant magnetic splitting inducing near-unity valley polarization in van der Waals heterostructures. Nat. Commun. 8, 1551 (2017).

26. Kiemle, J. et al. Control of the orbital character of indirect excitons in $\mathrm{MoS}_{2} /$ $W_{2}$ heterobilayers. Phys. Rev. B 101, 121404(R) (2020).

27. Jiang, C. Y. et al. Microsecond dark-exciton valley polarization memory in two-dimensional heterostructures. Nat. Commun. 9, 753 (2018).

28. Nagler, P. et al. Interlayer exciton dynamics in a dichalcogenide monolayer heterostructure. 2D Mater. 4, 025112 (2017).
29. Unuchek, D. et al. Room-temperature electrical control of exciton flux in a van der Waals heterostructure. Nature 560, 340-344 (2018).

30. Jauregui, L. A. et al. Electrical control of interlayer exciton dynamics in atomically thin heterostructures. Science $\mathbf{3 6 6}, 870-875$ (2019).

31. Fogler, M. M., Butov, L. V. \& Novoselov, K. S. High-temperature superfluidity with indirect excitons in van der Waals heterostructures. Nat. Commun. 5, 4555 (2014).

32. Wang, Z. F. et al. Evidence of high-temperature exciton condensation in twodimensional atomic double layers. Nature 574, 76-80 (2019).

33. Rivera, P. et al. Valley-polarized exciton dynamics in a $2 \mathrm{D}$ semiconductor heterostructure. Science 351, 688-691 (2016).

34. Unuchek, D. et al. Valley-polarized exciton currents in a van der Waals heterostructure. Nat. Nanotechnol. 14, 1104-1109 (2019).

35. Rivera, P. et al. Interlayer valley excitons in heterobilayers of transition metal dichalcogenides. Nat. Nanotechnol. 13, 1004-1015 (2018).

36. Yu, H. Y. et al. Moiré excitons: from programmable quantum emitter arrays to spin-orbit-coupled artificial lattices. Sci. Adv. 3, e1701696 (2017).

37. Komsa, H. P. \& Krasheninnikov, A. V. Electronic structures and optical properties of realistic transition metal dichalcogenide heterostructures from first principles. Phys. Rev. B 88, 085318 (2013).

38. Kang, J. et al. Electronic structural moiré pattern effects on $\mathrm{MoS}_{2} / \mathrm{MoSe}_{2} 2 \mathrm{D}$ heterostructures. Nano Lett. 13, 5485-5490 (2013).

39. Amin, B., Singh, N. \& Schwingenschlogl, U. Heterostructures of transition metal dichalcogenides. Phys. Rev. B 92, 075439 (2015).

40. Wang, $Y$. et al. Interlayer coupling in commensurate and incommensurate bilayer structures of transition-metal dichalcogenides. Phys. Rev. B 95, 115429 (2017).

41. Ruiz-Tijerina, D. A. \& Fal'ko, V. I. Interlayer hybridization and moire superlattice minibands for electrons and excitons in heterobilayers of transition-metal dichalcogenides. Phys. Rev. B 99, 125424 (2019).

42. Heo, H. et al. Interlayer orientation-dependent light absorption and emission in monolayer semiconductor stacks. Nat. Commun. 6, 7372 (2015).

43. Okada, M. et al. Direct and indirect interlayer excitons in a van der Waals heterostructure of hBN/WS $/ \mathrm{MoS}_{2} / \mathrm{hBN}$. ACS Nano 12, 2498-2505 (2018).

44. Kunstmann, J. et al. Momentum-space indirect interlayer excitons in transition-metal dichalcogenide van der Waals heterostructures. Nat. Phys. 14, 801-805 (2018).

45. Hanbicki, A. T. et al. Double indirect interlayer exciton in a $\mathrm{MoSe}_{2} \mathrm{WSe}_{2}$ van der Waals heterostructure. ACS Nano 12, 4719-4726 (2018).

46. Merkl, P. et al. Twist-tailoring Coulomb correlations in van der Waals homobilayers. Nat. Commun. 11, 2167 (2020).

47. Brem, S. et al. Hybridized intervalley moire excitons and flat bands in twisted WSe 2 bilayers. Nanoscale 12, 11088-11094 (2020).

48. Liu, G. B. et al. Electronic structures and theoretical modelling of twodimensional group-VIB transition metal dichalcogenides. Chem. Soc. Rev. 44, 2643-2663 (2015).

49. Tongay, S. et al. Tuning Interlayer coupling in large-area heterostructures with CVD-Grown MoS 2 and WS 2 monolayers. Nano Lett. 14, 3185-3190 (2014).

50. Liu, K. H. et al. Evolution of interlayer coupling in twisted molybdenum disulfide bilayers. Nat. Commun. 5, 4966 (2014).

51. van der Zande, A. M. et al. Tailoring the electronic structure in bilayer molybdenum disulfide via interlayer twist. Nano Lett. 14, 3869-3875 (2014).

52. Hong, X. P. et al. Ultrafast charge transfer in atomically thin $\mathrm{MoS}_{2} \mathrm{NS}_{2}$ heterostructures. Nat. Nanotechnol. 9, 682-686 (2014).

53. Ceballos, F. et al. Ultrafast charge separation and indirect exciton Formation in a $\mathrm{MoS}_{2}-\mathrm{MoSe}_{2}$ van der Waals heterostructure. ACS Nano 8, 12717-12724 (2014).

54. Jin, C. H. et al. Ultrafast dynamics in van der Waals heterostructures. Nat. Nanotechnol. 13, 994-1003 (2018).

55. Chen, H. L. et al. Ultrafast formation of interlayer hot excitons in atomically thin $\mathrm{MoS}_{2} \mathrm{NSS}_{2}$ heterostructures. Nat. Commun. 7, 12512 (2016).

56. Zhu, H. M. et al. Interfacial charge transfer circumventing momentum mismatch at two-dimensional van der Waals heterojunctions. Nano Lett. 17, 3591-3598 (2017).

57. Ji, Z. H. et al. Robust stacking-independent ultrafast charge transfer in $\mathrm{MoS}_{2} /$ WS $_{2}$ bilayers. ACS Nano 11, 12020-12026 (2017).

58. Wang, $\mathrm{K}$. et al. Interlayer coupling in twisted $\mathrm{WSe}_{2} \mathrm{WS}_{2}$ bilayer heterostructures revealed by optical spectroscopy. ACS Nano 10, 6612-6622 (2016).

59. Peng, B. et al. Ultrafast charge transfer in $\mathrm{MoS}_{2} / \mathrm{WSe}_{2} \mathrm{p}$-n heterojunction. $2 D$ Mater. 3, 025020 (2016). 
60. Pan, S. D. et al. Ultrafast charge transfer between $\mathrm{MoTe}_{2}$ and $\mathrm{MoS}_{2}$ monolayers. 2D Mater. 4, 015033 (2017).

61. Zereshki, P. et al. Interlayer charge transfer in $\operatorname{ReS}_{2} \mathbf{W S}_{2}$ van der Waals heterostructures. Phys. Rev. B 99, 195438 (2019).

62. Ceballos, F. et al. Highly efficient and anomalous charge transfer in van der Waals trilayer semiconductors. Nano Lett. 17, 1623-1628 (2017).

63. Ma, E. Y. et al. Recording interfacial currents on the subnanometer length and femtosecond time scale by terahertz emission. Sci. Adv. 5, eaau0073 (2019).

64. Zhu, X. Y. et al. Charge transfer excitons at van der Waals interfaces. J. Am. Chem. Soc. 137, 8313-8320 (2015).

65. Wang, $H$. et al. The role of collective motion in the ultrafast charge transfer in van der Waals heterostructures. Nat. Commun. 7, 11504 (2016).

66. Zhang, J. et al. Interlayer-state-coupling dependent ultrafast charge transfer in $\mathrm{MoS}_{2} \mathrm{WS}_{2}$ bilayers. Adv. Sci. 4, 1700086 (2017).

67. Long, R. \& Prezhdo, O. V. Quantum coherence facilitates efficient charge separation at a $\mathrm{MoS}_{2} / \mathrm{MoSe}_{2}$ van der Waals junction. Nano Lett. 16 1996-2003 (2016).

68. Li, L. Q., Long, R. \& Prezhdo, O. V. Charge separation and recombination in two-dimensional $\mathrm{MoS}_{2} \mathrm{WS}_{2}$ : time-domain ab initio modeling. Chem. Mater. 29, 2466-2473 (2017).

69. Tong, Q. J. et al. Topological mosaics in moire superlattices of van der Waals heterobilayers. Nat. Phys. 13, 356-362 (2017).

70. Zheng, Q. J. et al. Phonon-assisted ultrafast charge transfer at van der Waals heterostructure interface. Nano Lett. 17, 6435-6442 (2017).

71. Liu, F., Li, Q. Y. \& Zhu, X. Y. Direct determination of momentum-resolved electron transfer in the photoexcited van der Waals heterobilayer $\mathrm{WS}_{2} / \mathrm{MoS}_{2}$. Phys. Rev. B 101, 201405(R) (2020).

72. Liu, J. Y., Zhang, X. \& Lu, G. Excitonic effect drives ultrafast dynamics in van der Waals heterostructures. Nano Lett. 20, 4631-4637 (2020).

73. Qiu, D. Y., da Jornada, F. H. \& Louie, S. G. Optical spectrum of $\mathrm{MoS}_{2}$ : manybody effects and diversity of exciton states. Phys. Rev. Lett. 111, 216805 (2013).

74. Cheiwchanchamnangij, T. \& Lambrecht, W. R. L. Quasiparticle band structure calculation of monolayer, bilayer, and bulk MoS 2 . Phys. Rev. B 85, 205302 (2012).

75. Ramasubramaniam, A. Large excitonic effects in monolayers of molybdenum and tungsten dichalcogenides. Phys. Rev. B 86, 115409 (2012).

76. Komsa, H. P. \& Krasheninnikov, A. V. Effects of confinement and environment on the electronic structure and exciton binding energy of $\mathrm{MoS}_{2}$ from first principles. Phys. Rev. B 86, 241201(R) (2012).

77. Shi, H. L. et al. Quasiparticle band structures and optical properties of strained monolayer $\mathrm{MoS}_{2}$ and WS 2 . Phys. Rev. B 87, 155304 (2013).

78. Ye, Z. L. et al. Probing excitonic dark states in single-layer tungsten disulphide. Nature 513, 214-218 (2014).

79. Berkelbach, T. C., Hybertsen, M. S. \& Reichman, D. R. Theory of neutral and charged excitons in monolayer transition metal dichalcogenides. Phys. Rev. $B$ 88, 045318 (2013).

80. $\mathrm{Yu}, \mathrm{H}$. Y. et al. Anomalous light cones and valley optical selection rules of interlayer excitons in twisted heterobilayers. Phys. Rev. Lett. 115, 187002 (2015).

81. Meckbach, L. et al. Interlayer excitons in transition-metal dichalcogenide heterostructures with type-ll band alignment. J. Phys. Condens. Matter 30 374002 (2018).

82. Gillen, R. \& Maultzsch, J. Interlayer excitons in $\mathrm{MoSe}_{2} \mathrm{WSS}_{2}$ heterostructures from first principles. Phys. Rev. B 97, 165306 (2018).

83. Dong, $X$. Y. et al. Interlayer exciton-polaron effect in transition metal dichalcogenides van der Waals heterostructures. J. Phys. Chem. Solids 134, 1-4 (2019).

84. Latini, S. et al. Interlayer excitons and band alignment in $\mathrm{MoS}_{2} / \mathrm{hBN} / \mathrm{WS} \mathrm{S}_{2}$ van der Waals heterostructures. Nano Lett. 17, 938-945 (2017).

85. Van der Donck, M. \& Peeters, F. M. Interlayer excitons in transition metal dichalcogenide heterostructures. Phys. Rev. B 98, 115104 (2018).

86. Kamban, H. C. \& Pedersen, T. G. Interlayer excitons in van der Waals heterostructures: binding energy, Stark shift, and field-induced dissociation. Sci. Rep. 10, 5537 (2020).

87. Mouri, S. et al. Thermal dissociation of inter-layer excitons in $\mathrm{MoS}_{2} / \mathrm{MoSe}_{2}$ hetero-bilayers. Nanoscale 9, 6674-6679 (2017).

88. Ponomarev, E. et al. Semiconducting van der Waals interfaces as artificial semiconductors. Nano Lett. 18, 5146-5152 (2018).

89. Merkl, P. et al. Ultrafast transition between exciton phases in van der Waals heterostructures. Nat. Mater. 18, 691-696 (2019).
90. Li, L. H. et al. Wavelength-tunable interlayer exciton emission at the nearinfrared region in van der Waals semiconductor heterostructures. Nano Lett. 20, 3361-3368 (2020).

91. Yuan, L. et al. Twist-angle-dependent interlayer exciton diffusion in $\mathrm{WS}_{2}-\mathrm{WSe}_{2}$ heterobilayers. Nat. Mater. 19, 617-623 (2020).

92. Luong, D. H. et al. Tunneling photocurrent assisted by interlayer excitons in staggered van der Waals hetero-bilayers. Adv. Mater. 29, 1701512 (2017).

93. Gong, Y. J. et al. Vertical and in-plane heterostructures from $\mathrm{WS}_{2} / \mathrm{MoS}_{2}$ monolayers. Nat. Mater. 13, 1135-1142 (2014).

94. Heo, $\mathrm{H}$. et al. Rotation-misfit-free heteroepitaxial stacking and stitching growth of hexagonal transition-metal dichalcogenide monolayers by nucleation kinetics controls. Adv. Mater. 27, 3803-3810 (2015).

95. Karni, O. et al. Infrared interlayer exciton emission in $\mathrm{MoS}_{2} / \mathrm{WSe}_{2}$ heterostructures. Phys. Rev. Lett. 123, 247402 (2019).

96. Liu, Y. D. et al. Room temperature nanocavity laser with interlayer excitons in 2D heterostructures. Sci. Adv. 5, eaav4506 (2019).

97. Lee, C. H. et al. Atomically thin p-n junctions with van der Waals heterointerfaces. Nat. Nanotechnol. 9, 676-681 (2014).

98. Bellus, M. Z. et al. Tightly bound trions in transition metal dichalcogenide heterostructures. ACS Nano 9, 6459-6464 (2015).

99. Ceballos, F. et al. Probing charge transfer excitons in a MoSe $e_{2}-W_{2}$ van der Waals heterostructure. Nanoscale 7, 17523-17528 (2015).

100. Alexeev, E. M. et al. Imaging of interlayer coupling in van der Waals heterostructures using a bright-field optical microscope. Nano Lett. 17, 5342-5349 (2017).

101. Nayak, P. K. et al. Probing evolution of twist-angle-dependent interlayer excitons in $\mathrm{MoSe}_{2} \mathrm{NSe}_{2}$ van der Waals heterostructures. ACS Nano 11, 4041-4050 (2017).

102. Ross, J. S. et al. Interlayer exciton optoelectronics in a $2 \mathrm{D}$ heterostructure $\mathrm{p}-\mathrm{n}$ junction. Nano Lett. 17, 638-643 (2017).

103. Calman, E. V. et al. Indirect excitons and trions in $\mathrm{MoSe}_{2} \mathrm{WSe}_{2}$ van der Waals heterostructures. Nano Lett. 20, 1869-1875 (2020).

104. Schaibley, J. R. et al. Directional interlayer spin-valley transfer in twodimensional heterostructures. Nat. Commun. 7, 13747 (2016).

105. Surrente, A. et al. Defect healing and charge transfer-mediated valley polarization in $\mathrm{MoS}_{2} / \mathrm{MoSe}_{2} / \mathrm{MoS}_{2}$ trilayer van der Waals heterostructures. Nano Lett. 17, 4130-4136 (2017).

106. Paradisanos, I. et al. Controlling interlayer excitons in $\mathrm{MoS}_{2}$ layers grown by chemical vapor deposition. Nat. Commun. 11, 2391 (2020).

107. Kim, M. S. et al. Simultaneous hosting of positive and negative trions and the enhanced direct band emission in $\mathrm{MoSe}_{2} / \mathrm{MoS}_{2}$ heterostacked multilayers. ACS Nano 10, 6211-6219 (2016).

108. Cheng, R. et al. Electroluminescence and photocurrent generation from atomically sharp $\mathrm{WSe}_{2} / \mathrm{MoS}_{2}$ heterojunction $p-n$ diodes. Nano Lett. 14, 5590-5597 (2014).

109. Binder, J. et al. Upconverted electroluminescence via Auger scattering of interlayer excitons in van der Waals heterostructures. Nat. Commun. 10, 2335 (2019).

110. Wang, T. M. et al. Giant valley-zeeman splitting from spin-singlet and spintriplet interlayer excitons in $\mathrm{WSe}_{2} / \mathrm{MoSe}_{2}$ heterostructure. Nano Lett. 20 694-700 (2020).

111. Förg, M. et al. Cavity-control of interlayer excitons in van der Waals heterostructures. Nat. Commun. 10, 3697 (2019).

112. Yan, J. H. et al. Tunable control of interlayer excitons in $\mathrm{WS}_{2} / \mathbf{M o S}_{2}$ heterostructures via strong coupling with enhanced mie resonances. Adv. Sci. $\mathbf{6}$, 1802092 (2019).

113. Latini, S. et al. Cavity control of excitons in two-dimensional materials. Nano Lett. 19, 3473-3479 (2019).

114. Xia, J. et al. Strong coupling and pressure engineering in $\mathrm{WSe}_{2}-\mathrm{MoSe}_{2}$ heterobilayers. Nat. Phys. 17, 92-98 (2021)

115. Ubrig, N. et al. Design of van der Waals interfaces for broad-spectrum optoelectronics. Nat. Mater. 19, 299-304 (2020).

116. Wu, F. C., Lovorn, T. \& MacDonald, A. H. Theory of optical absorption by interlayer excitons in transition metal dichalcogenide heterobilayers. Phys. Rev. B 97, 035306 (2018).

117. Ciarrocchi, A et al. Polarization switching and electrical control of interlayer excitons in two-dimensional van der Waals heterostructures. Nat. Photonics 13, 131-136 (2019).

118. Kremser, M. et al. Discrete interactions between a few interlayer excitons trapped at a MoSe - WSe, heterointerface. npj 2D Mater. Appl. 4, 8 (2020). 
119. Li, W. J. et al. Dipolar interactions between localized interlayer excitons in van der Waals heterostructures. Nat. Mater. 19, 624-629 (2020).

120. Debnath, B. et al. Exciton condensate in bilayer transition metal dichalcogenides: strong coupling regime. Phys. Rev. B 96, 174504 (2017).

121. Cao, T. et al. Valley-selective circular dichroism of monolayer molybdenum disulphide. Nat. Commun. 3, 887 (2012).

122. Zeng, H. L. et al. Valley polarization in $\mathrm{MoS}_{2}$ monolayers by optical pumping. Nat. Nanotechnol. 7, 490-493 (2012).

123. Mak, K. F. et al. Control of valley polarization in monolayer $\mathrm{MoS}_{2}$ by optical helicity. Nat. Nanotechnol. 7, 494-498 (2012).

124. Sallen, $\mathrm{G}$. et al. Robust optical emission polarization in $\mathrm{MoS}_{2}$ monolayers through selective valley excitation. Phys. Rev. B 86, 081301(R) (2012).

125. Mak, K. F. et al. The valley Hall effect in $\mathrm{MoS}_{2}$ transistors. Science $\mathbf{3 4 4}$ 1489-1492 (2014).

126. Lee, J., Mak, K. F. \& Shan, J. Electrical control of the valley Hall effect in bilayer $\mathrm{MoS}_{2}$ transistors. Nat. Nanotechnol. 11, 421-425 (2016).

127. Lee, J. et al. Valley magnetoelectricity in single-layer MoS 2 . Nat. Mater. 16, 887-891 (2017).

128. Wu, S. F. et al. Electrical tuning of valley magnetic moment through symmetry control in bilayer $\mathrm{MoS}_{2}$. Nat. Phys. 9, 149-153 (2013).

129. Li, Y. L. et al. Valley splitting and polarization by the zeeman effect in monolayer MoSe 2 . Phys. Rev. Lett. 113, 266804 (2014).

130. Srivastava, A. et al. Valley Zeeman effect in elementary optical excitations of monolayer WSe, Nat. Phys. 11, 141-147 (2015)

131. Xiao, D., Yao, W. \& Niu, Q. Valley-contrasting physics in graphene: magnetic moment and topological transport. Phys. Rev. Lett. 99, 236809 (2007)

132. Yao, W., Xiao, D. \& Niu, Q. Valley-dependent optoelectronics from inversion symmetry breaking. Phys. Rev. B 77, 235406 (2008).

133. Xiao, D. et al. Coupled spin and valley physics in monolayers of $\mathrm{MoS}_{2}$ and other Group-VI dichalcogenides. Phys. Rev. Lett. 108, 196802 (2012).

134. Yu, H. Y., Liu, G. B. \& Yao, W. Brightened spin-triplet interlayer excitons and optical selection rules in van der Waals heterobilayers. 2D Mater. 5, 035021 (2018)

135. Zhang, L. et al. Highly valley-polarized singlet and triplet interlayer excitons in van der Waals heterostructure. Phys. Rev. B 100, 041402(R) (2019).

136. Huang, Z. M. et al. Robust room temperature valley hall effect of interlayer excitons. Nano Lett. 20, 1345-1351 (2020).

137. Hsu, W. T. et al. Negative circular polarization emissions from $\mathrm{WSe}_{2} / \mathrm{MoSe}_{2}$ commensurate heterobilayers. Nat. Commun. 9, 1356 (2018).

138. Wang, G. et al. Magneto-optics in transition metal diselenide monolayers. $2 D$ Mater. 2, 034002 (2015).

139. Li, Z. P. et al. Revealing the biexciton and trion-exciton complexes in BN encapsulated WSe. Nat. Commun. 9, 3719 (2018)

140. Li, Z. P. et al. Emerging photoluminescence from the dark-exciton phonon replica in monolayer WSe 2 . Nat. Commun. 10, 2469 (2019).

141. Seyler, K. L. et al. Signatures of moiré-trapped valley excitons in $\mathrm{MoSe}_{2} \mathrm{MSe}_{2}$ heterobilayers. Nature 567, 66-70 (2019).

142. Baek, $H$. et al. Highly energy-tunable quantum light from moiré-trapped excitons. Sci. Adv. 6, eaba8526 (2020).

143. Woźniak, $T$. et al. Exciton $g$ factors of van der Waals heterostructures from first-principles calculations. Phys. Rev. B 101, 235408 (2020)

144. Cao, Y. et al. Correlated insulator behaviour at half-filling in magic-angle graphene superlattices. Nature 556, 80-84 (2018).

145. Cao, Y. et al. Unconventional superconductivity in magic-angle graphene superlattices. Nature 556, 43-50 (2018)

146. Wu, F. C., Lovorn, T. \& MacDonald, A. H. Topological exciton bands in moiré heterojunctions. Phys. Rev. Lett. 118, 147401 (2017).

147. Zhang, N. et al. Moiré intralayer excitons in a $\mathrm{MoSe}_{2} / \mathrm{MoS}_{2}$ heterostructure. Nano Lett. 18, 7651-7657 (2018)

148. Lu, X. B., Li, X. Q. \& Yang, L. Modulated interlayer exciton properties in a twodimensional moire crystal. Phys. Rev. B 100, 155416 (2019).

149. Geng, W. T. et al. Moiré potential, lattice corrugation, and band gap spatial variation in a twist-free $\mathrm{MoTe}_{2} / \mathrm{MoS}_{2}$ heterobilayer. J. Phys. Chem. Lett. 11, 2637-2646 (2020).

150. Tran, K. et al. Evidence for moire excitons in van der Waals heterostructures. Nature 567, 71-75 (2019)

151. Jin, C. H. et al. Observation of moire excitons in $\mathrm{WSe}_{2} \mathrm{WS}_{2}$ heterostructure superlattices. Nature $\mathbf{5 6 7}, 76-80$ (2019).

152. Jin, C. H. et al. Identification of spin, valley and moire quasi-angular momentum of interlayer excitons. Nat. Phys. 15, 1140-1144 (2019).
153. Brotons-Gisbert, M. et al. Spin-layer locking of interlayer excitons trapped in moiré potentials. Nat. Mater. 19, 630-636 (2020).

154. Shimazaki, Y. et al. Strongly correlated electrons and hybrid excitons in a moire heterostructure. Nature 580, 472-477 (2020).

155. Alexeev, E. M. et al. Resonantly hybridized excitons in moiré superlattices in van der Waals heterostructures. Nature 567, 81-86 (2019).

156. Yu, H. Y. \& Yao, W. Electrically tunable topological transport of moiré polaritons. Sci. Bull. 65, 1555-1562 (2020).

157. Palummo, M., Bernardi, M. \& Grossman, J. C. Exciton radiative lifetimes in twodimensional transition metal dichalcogenides. Nano Lett. 15, 2794-2800 (2015).

158. Ovesen, S. et al. Interlayer exciton dynamics in van der Waals heterostructures. Commun. Phys. 2, 23 (2019).

159. Yu, Y. et al. Ultrafast Formation and dynamics of interlayer exciton in a largearea CVD-grown $\mathrm{WS}_{2} \mathrm{WSe}_{2}$ heterostructure. J. Phys. Condens. Matter 30, 495701 (2018).

160. Zhou, H. Z. et al. Controlling exciton and valley dynamics in two-dimensional heterostructures with atomically precise interlayer proximity. ACS Nano 14 4618-4625 (2020).

161. Choi, C. et al. Enhanced interlayer neutral excitons and trions in trilayer van der Waals heterostructures. npj 2D Mater. Appl. 2, 30 (2018).

162. Choi, J. et al. Moiré potential impedes interlayer exciton diffusion in van der Waals heterostructures. Sci. Adv. 6, eaba8866 (2020).

163. Wang, Q. S. et al. Valley carrier dynamics in monolayer molybdenum disulfide from helicity-resolved ultrafast pump-probe spectroscopy. ACS Nano 7, 11087-11093 (2013).

164. Mai, C. et al. Many-body effects in valleytronics: direct measurement of valley lifetimes in Single-Layer MoS. Nano Lett. 14, 202-206 (2014).

165. Zhu, C. R. et al. Exciton valley dynamics probed by Kerr rotation in WSe monolayers. Phys. Rev. B 90, 161302(R) (2014).

166. Hao, K. et al. Direct measurement of exciton valley coherence in monolaye WSe $e_{2}$ Nat. Phys. 12, 677-682 (2016)

167. Maialle, M. Z., de Andrada e Silva, E. A. \& Sham, L. J. Exciton spin dynamics in quantum wells. Phys. Rev. B 47, 15776-15788 (1993).

168. Yu, H. Y. et al. Dirac cones and Dirac saddle points of bright excitons in monolayer transition metal dichalcogenides. Nat. Commun. 5, 3876 (2014).

169. Yu, T. \& Wu, M. W. Valley depolarization due to intervalley and intravalley electron-hole exchange interactions in monolayer $\mathrm{MoS}_{2}$. Phys. Rev. B $\mathbf{8 9}$ 205303 (2014)

170. Jin, C. H. et al. Imaging of pure spin-valley diffusion current in $\mathrm{WS}_{2}-\mathrm{WSe}_{2}$ heterostructures. Science 360, 893-896 (2018).

171. Kim, J. et al. Observation of ultralong valley lifetime in $\mathrm{WSe}_{2} / \mathrm{MoS}_{2}$ heterostructures. Sci. Adv. 3, e1700518 (2017).

172. High, A. A. et al. Control of exciton fluxes in an excitonic integrated circuit. Science 321, 229-231 (2008).

173. Butov, L. V. Excitonic devices. Superlattices Microstruct. 108, 2-26 (2017).

174. Feldmann, J. et al. Linewidth dependence of radiative exciton lifetimes in quantum wells. Phys. Rev. Lett. 59, 2337-2340 (1987).

175. Deveaud, B. et al. Enhanced radiative recombination of free excitons in GaAs quantum wells. Phys. Rev. Lett. 67, 2355-2358 (1991).

176. Butov, L. V. et al. Condensation of indirect excitons in coupled AlAs/GaAs quantum wells. Phys. Rev. Lett. 73, 304-307 (1994).

177. Winbow, A. G. et al. Photon storage with nanosecond switching in coupled quantum well nanostructures. Nano Lett. 7, 1349-1351 (2007).

178. Hagn, M. et al. Electric-field-induced exciton transport in coupled quantum well structures. Appl. Phys. Lett. 67, 232-234 (1995).

179. Butov, L. V. \& Filin, A. I. Anomalous transport and luminescence of indirect excitons in AlAs/GaAs coupled quantum wells as evidence for exciton condensation. Phys. Rev. B 58, 1980-2000 (1998).

180. Butov, L. V. et al. Photoluminescence kinetics of indirect excitons in $\mathrm{GaAs} / \mathrm{Al}_{x} \mathrm{Ga}_{1-x}$ As coupled quantum wells. Phys. Rev. B 59, 1625-1628 (1999).

181. High, A. A. et al. Exciton optoelectronic transistor. Opt. Lett. 32, 2466-2468 (2007).

182. Butov, L. V., Gossard, A. C. \& Chemla, D. S. Macroscopically ordered state in an exciton system. Nature 418, 751-754 (2002).

183. Vörös, Z. et al. Long-distance diffusion of excitons in double quantum well structures. Phys. Rev. Lett. 94, 226401 (2005).

184. Ivanov, A. L. et al. Origin of the inner ring in photoluminescence patterns of quantum well excitons. Europhys. Lett. 73, 920-926 (2006). 
185. Rapaport, R., Chen, G. \& Simon, S. H. Nonlinear dynamics of a dense twodimensional dipolar exciton gas. Phys. Rev. B 73, 033319 (2006).

186. Chemla, D. et al. Room temperature excitonic nonlinear absorption and refraction in GaAs/AlGaAs multiple quantum well structures. IEEE J. Quantum Electron. 20, 265-275 (1984).

187. Szymanska, M. H. \& Littlewood, P. B. Excitonic binding in coupled quantum wells. Phys. Rev. B 67, 193305 (2003).

188. Grosso, G. et al. Excitonic switches operating at around 100 K. Nat. Photonics 3, 577-580 (2009).

189. Li, Z. D. et al. Exciton transport under periodic potential in MoSe2/WSe2 heterostructures. https://arXiv.org/2002.01561 (2020)

190. Woods, C. R. et al. Commensurate-incommensurate transition in graphene on hexagonal boron nitride. Nat. Phys. 10, 451-456 (2014).

191. Yoo, H. et al. Atomic and electronic reconstruction at the van der Waals interface in twisted bilayer graphene. Nat. Mater. 18, 448-453 (2019).

192. Liu, Y. W. et al. Tunable lattice reconstruction, triangular network of chiral one-dimensional states, and bandwidth of flat bands in magic angle twisted bilayer graphene. Phys. Rev. Lett. 125, 236102 (2020).

193. Zhang, S. et al. Abnormal conductivity in low-angle twisted bilayer graphene. Sci. Adv. 6, eabc5555 (2020)
194. Rosenberger, M. R. et al. Twist angle-dependent atomic reconstruction and Moiré patterns in transition metal dichalcogenide heterostructures. ACS Nano 14, 4550-4558 (2020).

195. Weston, A. et al. Atomic reconstruction in twisted bilayers of transition metal dichalcogenides. Nat. Nanotechnol. 15, 592-597 (2020).

196. Paik, E. Y. et al. Interlayer exciton laser of extended spatial coherence in atomically thin heterostructures. Nature 576, 80-84 (2019).

197. Lukman, S. et al. High oscillator strength interlayer excitons in twodimensional heterostructures for mid-infrared photodetection. Nat. Nanotechnol. 15, 675-682 (2020).

198. Mak, K. F. \& Shan, J. Photonics and optoelectronics of 2D semiconductor transition metal dichalcogenides. Nat. Photonics 10, 216-226 (2016).

199. Butov, L. V. Cold exciton gases in coupled quantum well structures. J. Phys. Condens. Matter 19, 295202 (2007).

200. Eisenstein, J. P. \& MacDonald, A. H. Bose-Einstein condensation of excitons in bilayer electron systems. Nature 432, 691-694 (2004).

201. Hsu, W. T. et al. Tailoring excitonic states of van der Waals bilayers through stacking configuration, band alignment, and valley spin. Sci. Adv. 5, eaax7407 (2019). 